\title{
EVIDENCE OF LIGHT-BENDING EFFECTS AND ITS IMPLICATION FOR SPECTRAL STATE TRANSITIONS
}

\author{
R. C. Reis ${ }^{1}$, J. M. Miller ${ }^{1}$, M. T. Reynolds ${ }^{1}$, A. C. Fabian ${ }^{2}$, D. J. Walton ${ }^{2,3}$, E. Cacketi ${ }^{4}$, and J. F. Steiner ${ }^{2}$ \\ ${ }^{1}$ Department of Astronomy, University of Michigan, Ann Arbor, MI 48109, USA \\ 2 Institute of Astronomy, University of Cambridge, Madingley Road, Cambridge CB3 OHA, UK \\ ${ }^{3}$ Space Radiation Laboratory, California Institute of Technology, Pasadena, CA 91125, USA \\ ${ }^{4}$ Department of Physics and Astronomy, Wayne State University, 666 W. Hancock Street, Detroit, MI 48201, USA \\ Received 2012 August 15; accepted 2012 November 22; published 2013 January 8
}

\begin{abstract}
It has long been speculated that the nature of the hard X-ray corona may be an important second driver of black hole state transitions, in addition to the mass accretion rate through the disk. However, a clear physical picture of coronal changes has not yet emerged. We present results from a systematic analysis of Rossi X-Ray Timing Explorer observations of the stellar-mass black hole binary XTE J1650-500. All spectra with significant hard X-ray detections were fit using a self-consistent, relativistically blurred disk reflection model suited to high ionization regimes. Importantly, we find evidence that both the spectral and timing properties of black hole states may be partially driven by the height of the X-ray corona above the disk, and related changes in how gravitational light bending affects the corona-disk interaction. Specifically, the evolution of the power-law, thermal disk, and relativistically convolved reflection components in our spectral analysis indicates that: (1) the disk inner radius remains constant at $r_{\text {in }}=1.65 \pm 0.08 \mathrm{GM} / \mathrm{c}^{2}$ (consistent with values found for the ISCO of XTE J1650-500 in other works) throughout the transition from the brighter phases of the low-hard state to the intermediate states (both the hard-intermediate and soft-intermediate), through to the soft state and back; (2) the ratio between the observed reflected X-ray flux and power-law continuum (the "reflection fraction," $R$ ) increases sharply at the transition between the hard-intermediate and soft-intermediate states ("ballistic" jets are sometimes launched at this transition); (3) both the frequency and coherence of the high-frequency quasi-periodic oscillations observed in XTE J1650-500 increase with $R$. We discuss our results in terms of black hole states and the nature of black hole accretion flows across the mass scale.
\end{abstract}

Key words: accretion, accretion disks - black hole physics - line: profiles - relativistic processes - X-rays: individuals (XTE J1650-500)

Online-only material: color figures

\section{INTRODUCTION}

The majority of stellar-mass black holes residing in lowmass binary systems spend most of their lives in quiescence. This explains the empirical fact that out of tens of thousands of such systems predicted to exist throughout our Galaxy (e.g., Yungelson et al. 2006), only about 50 have been discovered. For a number of such systems, it is well established that the outburst—which resulted in their discovery—evolves through a number of spectral states characterized by the relative strength of their thermal and non-thermal X-ray emission and with possible differences in the accretion geometry and reflection attributes. These active states can be roughly separated into four semi-distinct states which are phenomenologically described below and extensively discussed in Remillard \& McClintock (2006) and Belloni (2010). At the outset of the outburst, the system goes through what has been traditionally dubbed the low-hard state (LHS) where the X-ray spectrum is dominated by a non-thermal component often simply described by a power-law (photon index $\Gamma$ between $\sim 1.4-2$ ) spectrum with relatively low luminosity $\left(\sim 0.05 L_{\text {Edd }}\right.$ and exponential cutoff at $\sim 100 \mathrm{keV}$ ). The energy spectrum in the LHS peaks near $\sim 100 \mathrm{keV}$ and often we also see the weak presence of a thermal component (contributing $<20 \%$ of the total $2-20 \mathrm{keV}$ flux) with a temperature below $\sim 0.5 \mathrm{keV}$ produced by the accretion disk (see, e.g., Reynolds \& Miller 2011; Reis et al. 2010, and references therein).

As the luminosity increases, the spectrum moves through the intermediate state (IS) where the $2-10 \mathrm{keV}$ flux is typically a factor of $\sim 4$ times higher than that of the LHS. Here, the soft $(\Gamma=2-3)$ power-law tail coexists with a strong thermal component. Recently, the IS has begun to be subdivided into an early hard-intermediate state (HIS) and a later soft-intermediate state (SIS) just prior to a transition into the canonical high-soft state (HSS) or thermal state, where the X-ray flux is dominated ( $>75 \%$ of the total $2-20 \mathrm{keV}$ flux) by the thermal radiation from the inner accretion disk having an effective temperature of $\sim 1 \mathrm{keV}$. In this final state of the outburst, the system usually emits with luminosities $>0.1 L_{\text {Edd }}$ and the power-law component is both weak (less than $25 \%$ of the total $2-20 \mathrm{keV}$ flux) and steep $(\Gamma=2-3)$. Following the HSS, the system often returns to the LHS and subsequently goes back to quiescence where it can remain indefinitely or in some cases for a few years before this cycle restarts.

The hard X-ray emission predominant in the LHS has long been linked to inverse Compton scattering of the soft thermal disk photons by a population of hot $\left(\sim 10^{9} \mathrm{~K}\right)$ electrons in a cloud of optically thin, ionized gas or "corona" surrounding the inner parts of the accretion disk (Shapiro et al. 1976; Sunyaev \& Titarchuk 1980). Under the common assumption that the radio emission observed to originate from stellar-mass black holes is directly related to the presence of a jet, it is believed that all such systems, either in the LHS or in transition, launch a collimated outflow (e.g., Fender 2001; Fender et al. 2004, 2009). The fact that these persistent jets are observed only in the LHS suggests that the jet is linked to the corona, with claims that the corona in the LHS is indeed the launching point of persistent jets (see, e.g., Markoff et al. 2005). The connection between the radio (jet) and 
X-ray flux for both stellar-mass and supermassive black holes (Merloni et al. 2003; Gallo et al. 2003; Falcke et al. 2004), often referred to as "the fundamental plane" of black hole accretion, suggests an intimate connection between the corona and radio jets (see, e.g., Miller et al. 2012). Whether state transitions are driven by intrinsic changes in $\dot{m}$, physical changes in the disk, disk-corona, radio jet, or a combination of all these factors is a matter of much debate.

\subsection{Reprocessed X-Rays: Reflection}

The existence of a hard X-ray source-the corona-also adds further complexities to the various spectral states. The reprocessing of these hard X-rays by the relatively cold accretion disk in all active states results in a number of "reflection features" consisting of discrete atomic features together with a "Compton-hump" peaking at approximately $30 \mathrm{keV}$. The high fluorescent yield - and relatively high cosmic abundance-of iron often results in a particularly strong feature at $\sim 6-7 \mathrm{keV}$ (see, e.g., Fabian \& Ross 2010 for a recent review of "reflection" in black holes).

The strong irradiation of the black hole accretion disk by the coronal photons likely causes the surface layers to be photoionized. Ross \& Fabian (1993) investigated the effect of allowing the gas constituting the top layers of the accretion disk to ionize, and the authors went on to compute reflection spectra for different ionization levels. A number of similar studies of reflection from ionized matter have been conducted since (Matt et al. 1993, 1996; Ross et al. 1999; Nayakshin et al. 2000; Nayakshin \& Kallman 2001; Ballantyne et al. 2001; García \& Kallman 2010; García et al. 2011). These studies demonstrate that the reflection spectrum expected from a black hole depends strongly on the level of ionization of the surface layers of the disk. This can be quantified for a constant density gas by the ionization parameter

$$
\xi=\frac{L_{\mathrm{x}}}{n d^{2}},
$$

where $L_{\mathrm{x}}$ is the ionizing luminosity of the source, $d$ is the distance between the disk and the source, and $n$ is the density of the disk. Thus an increase in $\xi$, either by increasing the illuminating flux, decreasing the density or distance between the X-ray source and the disk, will cause the gas in the disk to become more ionized. Matt et al. $(1993,1996)$ split the behavior of the reflection spectrum into four main regimes depending on the value of $\xi$.

For low ionization parameter $\left(\xi<100 \mathrm{erg} \mathrm{cm} \mathrm{s}^{-1}\right)$, the material is only weakly ionized and the reflection spectrum resembles that arising from "cold" matter, with a prominent iron line at $6.4 \mathrm{keV}$, and strong absorption below $\approx 10 \mathrm{keV}$. There is only a weak contribution from the backscattered continuum at $\approx 6 \mathrm{keV}$ and a weak iron $\mathrm{K}$ absorption edge at $7.1 \mathrm{keV}$. As the disk becomes more ionized $\left(100<\xi<500 \mathrm{erg} \mathrm{cm} \mathrm{s}^{-1}\right)$ the system reaches intermediate ionization range where Fe has lost all of its M-shell $(n=3)$ electrons and thus exists in the form of Fe XVII-Fe XXIII with a vacancy in the $\mathrm{L}$ shell of the ion. Due to this vacancy, the $\mathrm{L}$ shell can absorb the $\mathrm{K} \alpha$ line photons and thus effectively trap the escaping photon. This resonance trapping is only terminated when an Auger electron is emitted. This second ionization regime is therefore characterized by a very weak iron line and a moderate iron absorption edge.

As the gas becomes more ionized $(500<\xi<$ $5000 \mathrm{erg} \mathrm{cm} \mathrm{s}^{-1}$ ) all low- $Z$ metals are found in their hydrogenic form and the soft reflection spectrum has only weak spectral features. Iron is found mostly in its hydrogen- or helium-like forms (Fe XXVI or Fe XXV, respectively) and due to the lack of at least two electrons in the L shell (i.e., not having a full 2 s sub-shell), Auger de-excitation cannot occur. The result is strong emission from "hot" $\mathrm{K} \alpha \mathrm{Fe}$ XXV and Fe XXVI at 6.67 and $6.97 \mathrm{keV}$, respectively, and the corresponding absorption edges at approximately 8.85 and $9.28 \mathrm{keV}$, respectively. Finally, when $\xi \gg 5000 \mathrm{erg} \mathrm{cm} \mathrm{s}^{-1}$, the disk is highly ionized and there is a distinct absence of any atomic features.

A further complication arises in the reflection spectra of stellar-mass black holes due to the fact that in these systems the gas in the accretion disk is inherently X-ray "hot" meaning that low- $Z$ metals can be fully ionized in the gas even before receiving any irradiation by the $\mathrm{X}$-ray corona. To account for this extra "thermal ionization," Ross \& Fabian (2007) performed self-consistent calculations of the reflection resulting from the illumination of the accretion disk by both a hard, power-law corona and thermal disk blackbody radiation. The authors compared the results of having the disk both in hydrostatic equilibrium and under the assumption of a constant density atmosphere, and found reasonably good agreement between the two emergent spectra. Ross \& Fabian (2007) also confirmed in stellar-mass black holes the result that had been previously found for active galactic nuclei (AGNs) in that the spectrum from a constant density disk is slightly diluted (it has a lower flux) in comparison to that of a disk in hydrostatic equilibrium. Furthermore, the authors also noted a few small differences between the modes; namely, a lower effective ionization parameter in the constant density model which resulted in a slightly stronger $\mathrm{FeK} \alpha$ line and deeper iron $\mathrm{K}$ edge. Nonetheless, the overall spectrum from the constant density approximation was shown to be in good agreement with the result for an atmosphere in hydrostatic equilibrium. The two reflection grids resulting from the work of Ross \& Fabian (2005, 2007) will be used frequently throughout this work.

\subsection{General Relativistic Effects: Light Bending}

Naively, assuming isotropic coronal emission, one would expect variations in the reflection component to be directly correlated to variations in the observed power-law continuum. That is, as the observed flux of the X-ray corona increases, so should the amount of reprocessed emission. However, in a number of instances it has been found that this is not the case, with the reflection component at times behaving in an anticorrelated manner (e.g., Rossi et al. 2005) or not varying at all despite large variations in the X-ray power-law continuum (e.g., Fabian et al. 2002; Fabian \& Vaughan 2003; Miniutti et al. 2003; Ballantyne et al. 2003; Larsson et al. 2007).

By virtue of its proximity to the black hole, the emission from the corona is naturally affected by general relativistic (GR) effects. Some of the radiation from the corona which would otherwise escape is gravitationally focused-"bent"-toward the accretion disk giving rise to enhanced reflection and selectively decreasing the X-ray continuum at infinity. A number of studies (for instance, Martocchia \& Matt 1996; Martocchia et al. 2000; Miniutti \& Fabian 2004; Miniutti et al. 2004; Niedźwiecki \& Życki 2008) have investigated the effect of GR on a compact, centrally concentrated X-ray corona close to a black hole. ${ }^{5}$ The "light-bending" model put forward by Miniutti \& Fabian (2004)

\footnotetext{
5 Observational evidence for such compact X-ray corona has recently come from microlensing results where the size of the X-ray-emitting region has been shown to be of the order of $\sim 10 r_{\mathrm{g}}$ (Chartas et al. 2009, 2012; Dai et al. 2010; Morgan et al. 2012).
} 
predicts a number of semi-distinct regimes affecting the variability of the reflection component compared to the X-ray continuum.

Regime 1. When the corona is very close to the black hole (a few gravitational radii $r_{\mathrm{g}}=G M / c^{2}$ ), a large fraction of the radiation is bent onto the accretion disk thus significantly reducing the amount of observed X-ray continuum and enhancing the reflection. A very steep emissivity profile is expected as the source is highly concentrated in the inner regions and the reflection is expected to be a steep function of the continuum in a quasi-linear manner.

Regime 2. When the central corona is slightly further from the black hole (at heights of $\sim 10 r_{\mathrm{g}}$ ), light bending causes the reflection component to vary significantly less than the $\mathrm{X}$-ray continuum. The amount of light bent toward the black hole decreases as the corona moves further from the black hole and the X-ray continuum increases.

Finally, at heights $\gg 20 r_{\mathrm{g}}$, light bending becomes less important and the observed continuum increases. ${ }^{6}$ In this manner, the presence of gravitational light bending has been invoked to explain the fact that Seyfert galaxies (e.g., Fabian et al. 2009, 2012b) and X-ray binaries (XRBs; e.g., Reis et al. 2012) at times appears to be "reflection dominated." Sources where the observed X-ray spectrum display a distinct lack (or comparably small amount) of hard, power-law-like continuum despite a large contribution of reflection are fully consistent with the first regime detailed above.

Of course, the model presented above is idealized in that all characteristics of the observed variabilities are assumed to be a result of variation in the height of an isotropic and compact corona with a fixed luminosity. Although intrinsic variation in the luminosity of the corona may well be present, it is unlikely that they could solely explain the behavior of the reflected emission described above. Indeed, the clear presence of broad and skewed iron emission lines in a growing number of sources ranging from stellar-mass black holes (Miller 2007; Miller et al. 2009b; Reis et al. 2009a; Steiner et al. 2011; Hiemstra et al. 2011) to AGNs (Tanaka et al. 1995; Nandra et al. 1997, 2007; Fabian et al. 2009; Brenneman et al. 2011; and also to lesser extent neutron stars (Cackett et al. 2008, 2010; di Salvo et al. 2005, 2009; Reis et al. 2009b)) strongly attest that GR effects play an important role in producing the line profile, further supporting the notion that the corona and the inner disk are both in the inner regions around a black hole.

\subsection{XTE J1650-500}

Among one of the first systems to provide observational evidence for the aforementioned effect of gravitational light bending around a black hole and indeed the first around a stellarmass black hole was XTE J1650-500, which was discovered by the Rossi X-Ray Timing Explorer (RXTE) on 2001 September 5 as it went into outburst (Remillard 2001). Based on the spectrum obtained early in decay of the outburst by XMM-Newton, and more importantly on the presence of a clearly broad iron emission line, Miller et al. (2002) were able to not only infer

\footnotetext{
6 In the original paper of Miniutti \& Fabian (2004) a further regime-Regime 3 -was defined at large radii where the reflection and power-law flux appeared to be anticorrelated with one another. This was an artifact of having a finite boundary for the disk extent of $100 r_{\mathrm{g}}$, and instead the reflected flux should asymptotically become flat with respect to the continuum as a continuation of Regime 2 (e.g., Niedźwiecki \& Życki 2008).
}

that the object in XTE J1650-500 was indeed a black hole, but also that it was close to maximally rotating with a dimensionless spin parameter $a \approx 0.998$.

By decomposing the hard X-ray continuum from the reflection component in three BeppoSAX observations of XTE J1650-500, Miniutti et al. (2004) were able to show that the latter remained nearly constant despite a large change in the direct continuum, in a manner consistent with the predictions of light bending around a black hole. Optical observations obtained after the system had returned to near quiescence (Orosz et al. 2004) revealed a mass function $f(M)=2.73 \pm 0.56 M_{\odot}$ with a most likely mass of $\sim 4 M_{\odot}$, and with it secured XTE J1650-500 as genuine black hole binary system. Corbel et al. (2004) reported on the radio and X-ray observations of XTE J1650-500 during the outburst. The authors found a clear drop by nearly an order of magnitude in the radio flux at the transition from the HIS (referred to as the IS in that work) to the SIS (referred to as the steep power-law state in that work), and surprisingly they found residual radio emission during the often radio-quiet disk-dominated soft state, which they attributed to possible emission from previously ejected material interacting with the interstellar medium (ISM), rather than originating in the central source.

A follow-up study of RXTE data by Rossi et al. (2005) used the iron line as a proxy to the total reflection component and confirmed the plausibility of the light-bending scenario for the evolution of XTE J1650-500. Again using data obtained from the $R X T E$, Homan et al. (2003) reported on the discovery of a $\sim 250 \mathrm{~Hz}$ quasi-periodic oscillation (QPO) together with a number of less coherent variability peaks at lower frequencies. By studying the spectral and timing evolution during the first $\sim 80$ days of the outburst, the authors were able to define six periods (I-VI, in this work referred to as P1-P6) having somewhat distinct spectral and timing characteristics (see their Figure 1 and Table 1). A recent study involving XTE J1650-500 has discussed the similarities between X-ray binaries and AGNs (Walton et al. 2012) and argued that both XTE J1650-500 and the active galaxy MCG -6-30-15 (Tanaka et al. 1995) must contain a rapidly rotating black hole, with the spin of XTE J1650-500 having been formally constrained to $0.84 \leqslant a \leqslant$ 0.98 . A further body of work based on RXTE observations and a variety of empirical models for the hard X-ray continuum (Yan $\&$ Wang 2012) has concluded that the emission region, here referred to as the corona, decreases by a factor of $\sim 23$ in size during the transition from the hard to the soft state.

\subsection{This Work}

Since its discovery, XTE J1650-500 has become one of the best-studied black hole systems. However, the energy spectra of this system have either been studied in a great degree of detail using high-quality, single snapshot observations with XMM-Newton (i.e., Miller et al. 2002; Walton et al. 2012) or BeppoSAX (Miniutti et al. 2004) or using mostly phenomenological and simple models in the study of long-term evolutions with RXTE (i.e., Rossi et al. 2005; Dunn et al. 2010, 2011; Yan $\&$ Wang 2012). In this paper, we use the full RXTE archival data of the outburst to investigate the evolution of the direct power-law continuum, reflection, and thermal disk components using, for the first time, a fully self-consistent prescription for the reflection component.

In this manner, we combine the virtues of detailed analyses of single observations with the immense diagnostic power of multiple RXTE pointings. By being able to decouple the total 

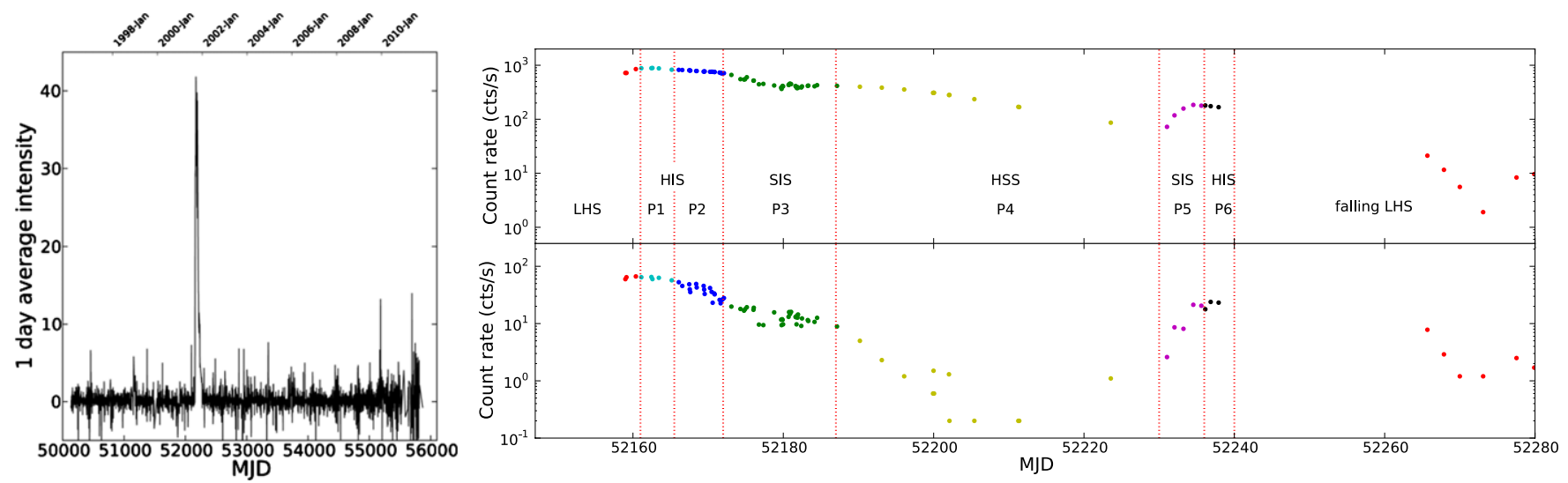

Figure 1. The 181 observations used were taken during the 2001/2002 outburst which is clearly seen in the all-sky monitor (left). Since its discovery outburst, XTE J1650-500 has remained in quiescence. Right: PCA-PCU2 (top) and HEXTE-A (bottom) count rate during the time encompassing the outburst. The different colors mark the distinct spectral states as defined by Homan et al. (2003) based on the timing characteristics of the source.

(A color version of this figure is available in the online journal.)

reflection component $(\mathrm{Fe}-\mathrm{K} \alpha$ emission line together with all other reflection signatures) from the illuminating continuum, we find that the transition from the HIS to the SIS is accompanied by a sharp increase in the strength of the reflected emission in comparison to the direct continuum. We interpret this increase in the reflection fraction as a sudden collapse of the corona as the system approaches the thermal state, although we note that this may not be a unique interpretation.

This paper is structured as follows. Section 2 briefly introduces the observations and details our various selection criteria. Section 3 describes the base model and assumptions used throughout this work. The various results are presented in Section 4 and in Section 5 we present a qualitative picture of a possible physical scenario that combines all our findings.

\section{OBSERVATION AND DATA REDUCTION}

We downloaded and analyzed all 181 individual RXTE pointed observations of XTE J1650-500 following its discovery. This gave a total of 307.4 ks Proportional Counter Array (PCA) exposure which were reprocessed with the latest HEASOFT ver. 6.12 tools. As such, we followed all the well established, standard reduction guides. ${ }^{7}$ Given that it is the only Proportional Counter Unit (PCU) that is always on, as well as being the best calibrated of all units, we used only data from PCU-2. We chose to use the standard offset value of $<0.02$ as well as elevation above Earth's limb $>10^{\circ}$. Background spectra were produced using PCABACKEST using filter files created using XTEFILT. The latest PCA-history file was also used and throughout this work we use PCA data between $3 \mathrm{keV}$ (ignored channels $\leqslant 6$ ) and $25 \mathrm{keV}$ without additional systematic errors as we are mostly interested in relative changes and the impact on the errors of the various parameters as well as in the $\chi^{2}$ distribution shown in Figure 6 is minimal with the only change being a systematic shift to lower values.

For our analyses, we require both PCA and High Energy $X$-Ray Timing Experiment (HEXTE) data (but see below). The HEXTE data were also reduced in the standard manner following the procedures outlined in the RXTE guide. Background files were generated using HXTBACK and the spectra were corrected for dead time using HXTDEAD. The appropriate response and

\footnotetext{
7 Found at http://heasarc.nasa.gov/docs/xte/recipes/cook_book.html.
}

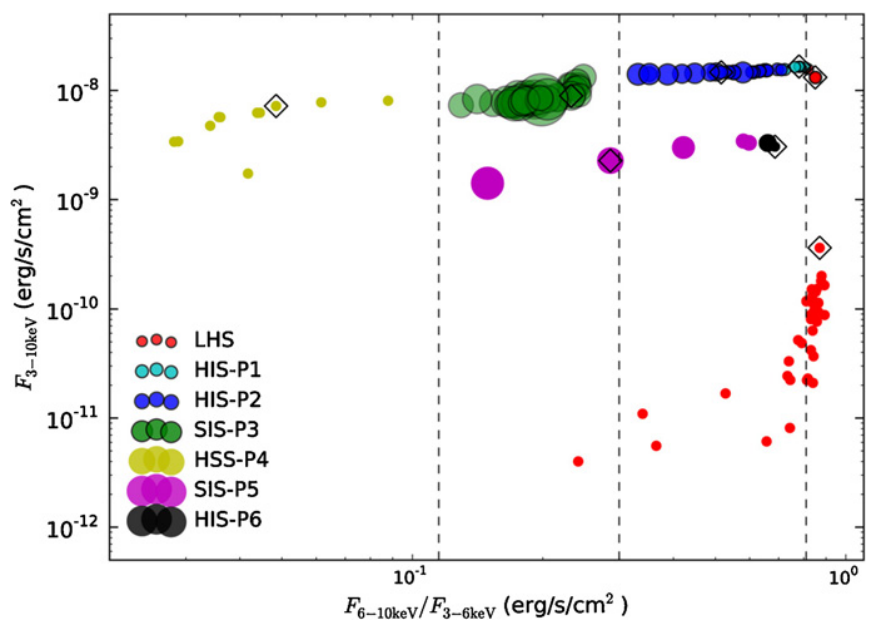

Figure 2. Hardness intensity diagram made using the 116 observations that remain after imposing a cut where both HEXTE Clusters A and B have sourcebackground counts greater than 0 . The vertical dashed lines mark approximate transitions between LHS-HIS-SIS-HSS. The large diamond symbols mark the position in the HID diagram for the representative spectra shown in Figure 4. The color code is identical to Figure 1 (right) and will remain the same throughout this work. We have scaled the size of the symbols for each observation to the value of the reflection fraction $R$, as determined in Section 4.1 and shown in Figure 3. The size legends from top to bottom are: $R=0.5,0.75,1,2,3,4,5$. The falling branch of the LHS and the HSS has sizes corresponding to $R=0.5$ as $R$ is poorly constrained in these states.

(A color version of this figure is available in the online journal.)

ancillary files were downloaded. ${ }^{8}$ HEXTE data were fit between 25 and $150 \mathrm{keV}$.

Figure 1 (left) shows the 1 day averaged long-term light curve as seen from the RXTE all-sky monitor (ASM) where the 2001 outburst is clearly visible. The panels on the right shows the PCA-PCU2 count rate (top) and HEXTE-A (bottom) during the time roughly encompassing the outburst. The colors highlight the various states during the outburst and are directly mapped into the hardness intensity diagram (HID) shown in Figure 2. In short, the first few observations caught the source in a rising LHS which evolved to the HIS approximately 3 days later, where it remained for $\sim 15$ days until the clear change to the SIS. We have further divided the HIS into early Period 1 and late Period 2,

8 From ftp://legacy.gsfc.nasa.gov/xte/calib_data/hexte_files/DEFAULT/. 
similar to the division made by Homan et al. (2003) based on the timing characteristics displayed. The source then remains in the SIS (P3) for approximately 12 days before it makes the typical excursion to the disk dominated HSS (P4) where we see a clear drop in the HEXTE count rate in Figure 1. After $\sim 40$ days, the hard flux in XTE J1650-500 sharply increases (P5 and P6) as the system returns to LHS and eventually goes back into quiescence where it remains up to the present time.

The work presented in this paper is fully interpreted within the framework of reflection models (see, e.g., Fabian \& Ross 2010; and Section 1.1). As such we are mainly interested in being able to determine the contribution of the reflected emission to the overall spectra. As mentioned in the introduction, reflection is not limited to the iron line profile and in order to obtain the best handle on the full reflection component we restricted the analysis that follows by requiring that both the HEXTE-A and the HEXTE-B units have background-subtracted count rates that are greater than zero. This results in 116 PCA pointing observations totaling $217.9 \mathrm{ks}$. In doing so, we are effectively reducing our sensitivity to observations in very steep or disk-dominated states, as well as to faint LHS, and most of the results presented here concern the ISs, which are more luminous at high energies (see Figure 1, right). In order to investigate the effect of the HEXTE data on the results presented in this paper, we have also repeated all the fits described below using only the PCA data and confirmed that, although the results obtained do not strictly depend on the inclusion of the HEXTE data, owing to its relatively small statistical weight, the high-energy data still provide a useful additional test of the continuum model (see, e.g., Figure 4).

In the following section we detail the spectral fits to the 116 observations. All our work makes use of the X-ray fitting package XSPEC version 12.7.0 (Arnaud 1996). Where uncertainties on model parameters are quoted, this refers to the $90 \%$ confidence limit for the parameter of interest.

\section{THE MODEL}

Previous attempts to characterize the evolution of XTE J1650-500 as observed with $R X T E$ have relied on a purely phenomenological interpretation of the reflection continuum and features 9 and often employed a single "Gaussian" emission line with centroid energy fixed at $6.4 \mathrm{keV}$ as expected from neutral Fe-K $\alpha$ (i.e., Dunn et al. 2011), a combination of a similar "Gaussian" together with a broad smeared edge (SMEDGE; Ebisawa et al. 1994) component (i.e., Yan \& Wang 2012), or in the more physically appropriate application, a relativistic emission line (LAOR; Laor 1991) together with SMEDGE (Rossi et al. 2005). However, even in the latter example, the model was unphysical as the combination of SMEDGE with an emission line does not maintain consistency between the depth of the edge - the number of absorbed photons - and the strength of the line.

In the study of Dunn et al. (2011), where the authors were primarily interested in the behavior of the thermal accretion disk,

\footnotetext{
9 Contrast this phenomenological approach to the reflection component with the systematic testing of Comptonization models for the power-law (corona) component by Nowak et al. (2002) on RXTE data of GX 339-4. Ideally, one would strive to combine physical models for both the power law and reflection (and of course the accretion disk which is known to not be a simple multicolor disk due to various relativistic effects); however, this quest for fully relativistic disk and reflection together with physical prescription for the Comptonization continuum is proving highly complicated even for single, dedicated efforts at snapshot observations and is beyond the scope of this paper.
}

such a simplification is easily justified as small deviations at high energies are unlikely to have significant effects at low energies. Rossi et al. alluded to the importance of using reflection models but unfortunately were swayed away due to the highly time-consuming and computer-intensive task that this would have presented nearly 10 years ago.

The importance of reflection from accretion disks can be directly measured by the sheer number of theoretical works that have been devoted to fully characterizing its behavior (i.e., Lightman \& White 1988; George \& Fabian 1991; Matt et al. 1991; Ross \& Fabian 1993, 2005, 2007; Zycki et al. 1994; Nayakshin et al. 2000; Ballantyne et al. 2001; García \& Kallman 2010; García et al. 2011). A widely used reflection code is that of Ross \& Fabian (2005; REFLIONX), which provides a self-consistent treatment of the dominant atomic processes around a black hole and, given an input power-law continuum, outputs a reflection spectrum where both the "Compton-hump," emission and absorption features are all physically linked.

The reflection spectrum is calculated in the local frame, therefore we employ the convolution model KDBLURf (Laor 1991; vastly optimized by Jeremy S. Sanders and employed in Fabian et al. 2012a; Reis et al. 2012) to model the relativistic effects in the spectra. Despite the existence of newer relativistic models that includes the black hole spin (as opposed to the extent of the inner radius) as a variable parameter (i.e., Beckwith \& Done 2004; Dovčiak et al. 2004; Brenneman \& Reynolds 2006; Dauser et al. 2010) the advantage of using KDBLURf on such a vast data set is that it greatly expedites the fits and since we are not overly interested in absolute values, the small improvements of the newer models at the $10 \%$ level (Beckwith \& Done 2004) do not justify their use.

Each of the 116 remaining spectra were thus fit with a base model described in XSPEC as ${ }^{10}$

$$
\begin{aligned}
\text { PHABS } \times & (\text { POWERLAW + DISKBB } \\
& + \text { KDBLURf } \otimes \text { REFLIONX })
\end{aligned}
$$

To avoid unnecessary CPU cost, all spectra were fitted adopting the same initializing values for the model parameters. A similar approach of using a base model with similar starting parameters was taken by Wilms et al. (2006)—among others_-in the study of Cygnus X-1.

Since the low-energy cutoff in the PCA of $\sim 3 \mathrm{keV}$ is not sufficient to constrain the neutral hydrogen column density, throughout this work we have frozen the parameter in the PHABS model to $7 \times 10^{21}$ atom $\mathrm{cm}^{-2}$ in XSPEC as the neutral column density is likely best modeled as being constant with time (Miller et al. 2009a). ${ }^{11}$ The key parameters in the REFLIONX model are the spectral shape of the illuminating continuum, which is set to be the same as that of the direct power law and the ionization parameter of the accretion disk (as in Equation (1)). The iron abundance was set to the solar value and the redshift was set to zero.

\footnotetext{
10 We have also repeated this experiment with the latest reflection model by Ross \& Fabian (2007; REFBHB; see also Reis et al. (2008) for a description of its use) which incorporates a blackbody component and found the results to be consistent in both cases. We choose to carry on with the

REFLIONX + DISKBB combination for a number of reasons; to start, this combination can be easily reproduced (REFBHB is not yet public) and the output parameters are somewhat standardized. For example, REFLIONX has $\xi$ as a free parameter whereas REFBHB has the more obscure combination of hydrogen number density $n_{\mathrm{H}}$ and the relative strength of the illuminating flux over the thermal emission at the surface of the disk.

11 We used the standard BCMC cross-sections (Balucinska-Church \& McCammon 1992) and ANGR abundances (Anders \& Grevesse 1989).
} 
In order to make the work more tractable, the emissivity profile in the blurring component was initially restricted to the form of a single power law such that $\varepsilon(r) \propto r^{-q}$, and following the most recent work on the best XMM-Newton and BeppoSAX data of XTE J1650-500 (Walton et al. 2012) we have frozen the inclination of the accretion disk to $65^{\circ}$ and note that this parameter is highly constrained based on optical light curve to be greater than $50^{\circ} \pm 3^{\circ}$ and $<80^{\circ}$ (Orosz et al. 2004). We also check whether or not the results presented below change if we freeze the inclination at this lower limit $\left(50^{\circ}\right)$ and we confirm that they do not. As is standard with such fits, we have frozen the outer radius to the maximum value in the grid of $400 r_{\mathrm{g}}$ and the inner accretion disk is free to vary.

A phenomenological combination of an LAOR emission line on top of a reflection model such as PEXRIV (Magdziarz \& Zdziarski 1995)—which does not include the iron-K $\alpha$ emission line-often provides an equally good fit to X-ray spectra of black holes. However, PEXRIV does not Compton broaden its absorption edge nor does it provide a physical coupling between itself and the extra LAOR component which can result in parameters severely lacking in physical consistency. This is exemplified in Reis et al. (2012) where we showed for the stellar-mass black hole candidate MAXI J1836-194 that the reflection component could be equally well modeled with a combination of LAOR + PEXRIV; LAOR + KDBLUR $\times$ PEXRIV; or $\mathrm{KDBLUR} \times \mathrm{REFLIONX}$. The first combination unphysically required the iron line to be coming from far within $6 r_{\mathrm{g}}$ in the strong gravity regime, yet all other reflection features - under the formalization of the model - appeared exempt from such effects. After properly correcting for relativistic effects in PEXRIV, the ionization parameter $\xi$ of the second model was nearly two orders of magnitude higher than the previous combination as the Fe absorption edge in PEXRIV was no longer trying to fit the blue wing of the iron line. This change was also accompanied by an artificial hardening of the power-law index and a decrease in the equivalent width of the LAOR component from $\sim 270 \mathrm{eV}$ to $\sim 180 \mathrm{eV}$. Correctly blurring the originally sharp absorption edge caused it to become smooth and more symmetric. Due to the decoupling between the LAOR line and PEXRIV, this smooth edge traded off with the LAOR line component, thus decreasing the equivalent width of the latter. As such, we stress the importance of the imposed physical consistency in the emission line and corresponding absorption edge strength afforded by REFLIONX and strictly enforce this in the work that follows.

\section{RESULTS AND DISCUSSION}

\subsection{Evolution of the Outburst}

Figure 3 shows the evolution of all the parameters of interest in this work (see Table 2), ${ }^{12}$ and Figure 4 shows the best fit to eight representative spectra roughly covering the eight periods highlighted in Figures 1 and 2 and described in detail in Homan et al. (2003). The spectra used for illustration are shown in Figure 2 with diamonds. The top four panels in Figure 3 show the evolution of the extrapolated $0.1-1000 \mathrm{keV}$ fluxes for the total, POWERLAW, REFLIONX, and DISKBB components, from top to bottom, respectively. All fluxes were obtained using

\footnotetext{
12 We refer the reader to the work of Dunn et al. (2011) for the evolution of the disk properties during the outburst. As mentioned above, despite the fact that the authors did not correctly account for reflection in their work, this plays a minor role at the energies of interest in regards to the disk properties and as such that work is still a valid and important reference for the evolution of accretion disks.
}

CFLUX in XSPEC. The vertical dotted lines running through all the panels highlight the eight periods shown in Figures 1 and 2. We see a clear increase in the disk flux during the first $\sim 30$ days followed by a clear flattening as it moves into the HSS. It is also visually apparent that the reflection flux varies relatively less than the power-law continuum. This will be investigated further in what follows.

The following two panels show the evolution of the disk temperature and ionization parameter, $\xi$. Early in the outburst, the disk was relatively cold $(\lesssim 0.5 \mathrm{keV})$ and only moderately ionized with $\xi \approx 200 \mathrm{erg} \mathrm{cm} \mathrm{s}^{-1}$. As the system moves into the HSS, the ionization increases smoothly until $\sim 2$ days before the transition to the SIS when $\xi$ sharply increases to $\xi \approx 3000 \mathrm{erg} \mathrm{cm} \mathrm{s}^{-1}$ and remains at that level through the transition up to the end of the SIS. The disk temperature, on the other hand, appears to reach a relativity stable value of $\sim 0.6-0.65 \mathrm{keV}$ early in P2, approximately halfway through the HIS. As the system progress into the HSS, the disks become fully ionized with $\xi$ reaching the maximum allowed value in the model $(\log \xi=4)$, before coming back down to the low hundreds toward the end of the outburst.

The reflection fraction $R$ shown in the third from bottom panel of Figure 3 is here defined as a measure of the ratio between the (observed) continuum power law to the reflection flux emitted by the disk. Since a fraction of the power-law illuminating the accretion disk is downscattered as it is reprocessed in the disk, the reflection fraction in REFLIONX is calculated by dividing the extrapolated ( $1 \mathrm{eV}-1000 \mathrm{keV})$ REFLIONX flux by the $0.1-1000 \mathrm{keV}$ power-law flux. At the start of the outburst, through to the end of the HIS, $R$ increases smoothly between $\approx 0.6-1$. At the transition between the HIS to the SIS, $R$ displays a sharp increase to $\approx 4$ where it remains until the beginning of the disk-dominated HSS where the power law effectively disappears. We also show in this panel the ratio of the $3-100 \mathrm{keV}$ reflection to power-law flux. The behavior described above is still qualitatively the same and we still see a clear jump in this ratio at the transition from the HIS to the SIS. However, when limited to the $3-100 \mathrm{keV}$ flux, this ratio is systematically a factor of $\approx 1.5$ less than the extrapolated ratio; a direct result of not accounting for the extra downscattered flux at low energies.

As a further test of both the qualitative (clear jump in $R$ between HIS and SIS) as well as quantitative (change from $R \approx 0.6$ to $R \approx 4$ between $\mathrm{P} 1$ and $\mathrm{P} 3$ ) behaviors found here for the reflection fraction, we temporally replace the REFLIONX model with a combination of LAOR plus PEXRIV and employ this model to the observations highlighted in Figures 2 and 4 for P1 and P3. In using this model, we have blurred the PEXRIV component with the same parameters as the LAOR line profile. Figure 5 shows the confidence range for the reflection fraction (a free parameter of PEXRIV) for these two representative spectra. In agreement with our previous results, we see that in the HIS the reflection fraction is constrained to $R=0.58_{-0.11}^{+0.08}$ and in the SIS it is $R>2.7$ at the $90 \%$ level of confidence $\left(\Delta \chi^{2}=2.71\right)$.

The fact that $\xi$ is maximal in the HSS (P4) despite the fact that this is when the irradiation appears to be at its lowest level (second panel from the top) can be explained with a number of scenarios. We showed in Section 1.1 that in stellar-mass black holes the intrinsic hot disk can result in significant thermal ionization (Ross \& Fabian 2007) which will be strongest in the disk-dominated HSS. Thus, in this scenario, the high ionization measured could also be in part due to thermal ionization. If this thermal component is the sole source of ionization in the HSS, $R$ would indeed go to zero. A further possibility is that the disk is 


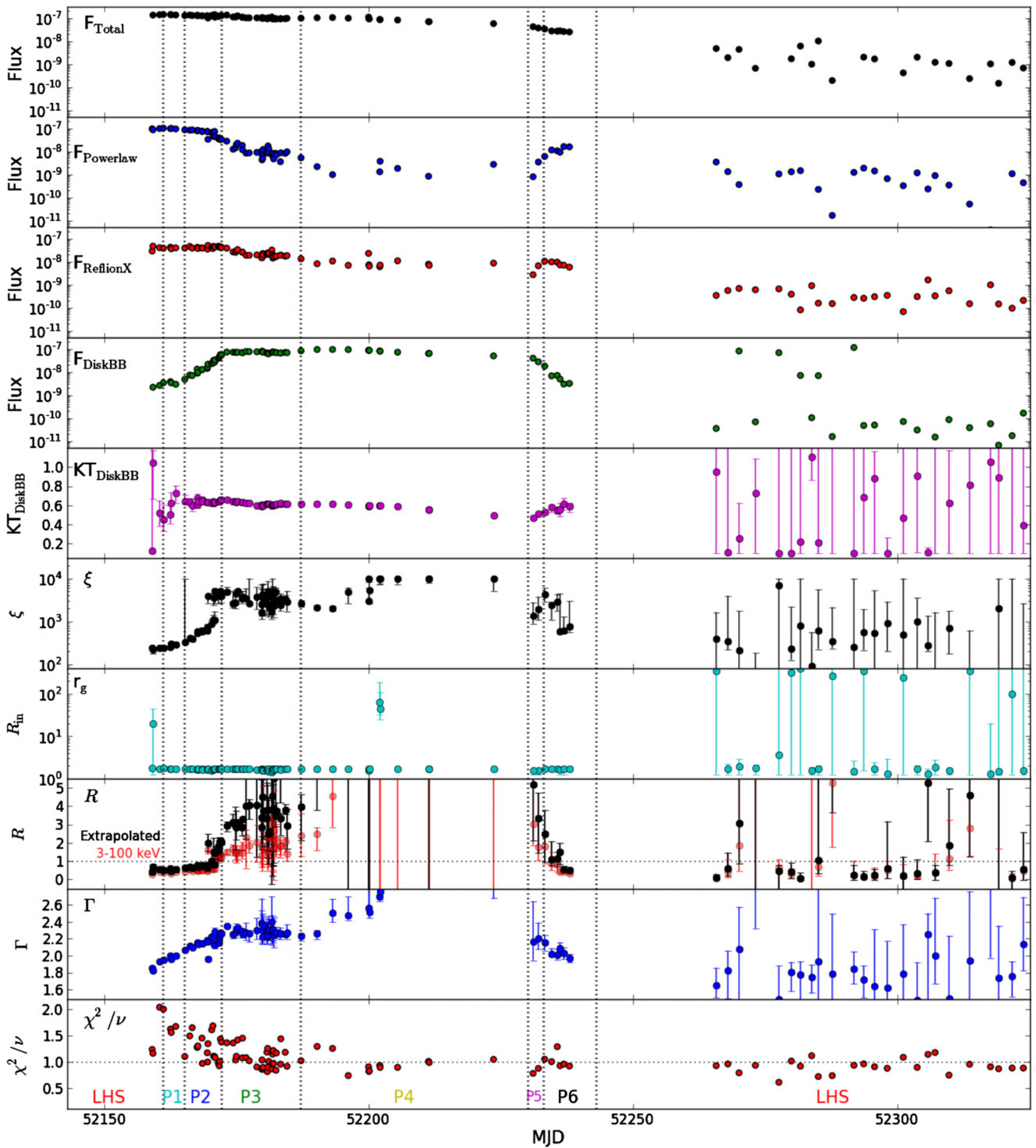

Figure 3. Top four panels: extrapolated $0.1-1000 \mathrm{keV}$ flux evolution for the total, power law, reflection, and disk components. The errors in the fluxes were assessed using CFLUX but are omitted here for clarity (but see Figure 7 for an indication of the magnitude of the errors in each state). These errors were propagated in the derivation of the reflection fraction $R$ (eighth panel from the top). We also show in the eighth panel $(R)$ the ratio of the $3-100 \mathrm{keV}$ reflection to power-law fluxes. A jump is in this ratio seen at the same position as that for the extrapolated fluxes. The bottom panel shows the reduced- $\chi^{2}$ for 114 dof. The vertical dashed lines show the transition from the rising LHS, P1-P6 and back into the falling LHS.

(A color version of this figure is available in the online journal.)

indeed highly photoionized as a result of strong focusing of the coronal photons onto the disk. This would significantly remove the number of hard photons escaping the system (thus explaining the second panel from the top) and cause the disk to be highly ionized. Indeed, observations of disk winds originating in the HSS of various black hole binaries (BHBs) consistently show winds having $\xi \sim 10^{4} \mathrm{erg} \mathrm{cm} \mathrm{s}^{-1}$ (e.g., Miller et al. 2008a; Neilsen \& Lee 2009; King et al. 2012; Ponti et al. 2012).
Unfortunately, the lack of reliable constrains on the reflection fraction in the HSS prevents us from making any solid claims on the nature of the disk-corona interaction in this state.

\subsection{Disk Emissivity}

In Figure 6 (left) we show the distribution of the reduced- $\chi^{2}$ (for 115 degrees of freedom) from all observations assuming the simple Newtonian "lamp-post"-like geometry in which the 

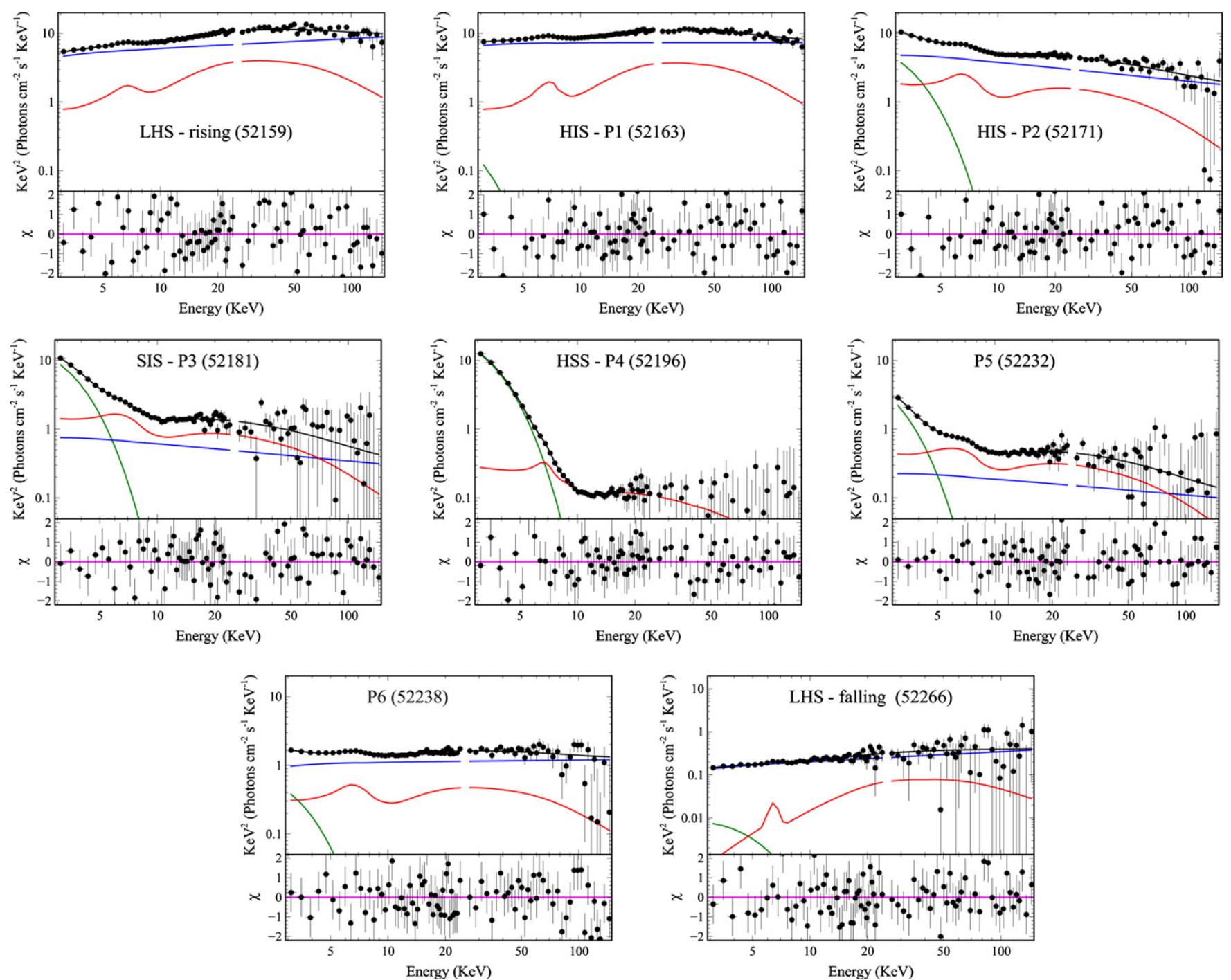

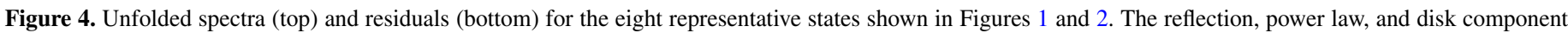

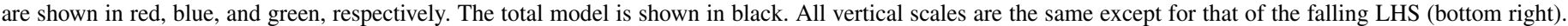
The approximate MJD of each observation is shown in brackets.

(A color version of this figure is available in the online journal.)

emissivity profile follows a $q=3$ power law (light blue), as well as after relaxing this assumption (red). In both cases there is a clear peak at reduced- $\chi^{2}=1$; however, this peak is much more distinct upon relaxing the Newtonian approximation. The Newtonian approximation naturally does not take into account the effects of general relativity that will be experienced by the emission from the corona and accretion disk near the black hole. Relativistic effects (strong gravity as well as relativistic time dilation) act to steepen the emissivity in the inner regions of the disk. The right panels of Figure 6 show either the distribution of the emissivity index (blue) or the $90 \%$ lower limit in their value (red) for the spectral states indicated in each panel. We refer the reader to the work of Miniutti \& Fabian (2004), Wilkins \& Fabian (2011, 2012), Fabian et al. (2012a), and references therein for a detailed study examining non-Newtonian values for the emissivity index, but note here that steep emissivities similar to those found here for the HIS and SIS are a natural and unavoidable consequence of strong gravity.

The bottom panel of Figure 6 is used here as a simple illustration of the evolution in $q$ as well as the count rate in both the PCA and HEXTE data. It is clear that $q$ can only

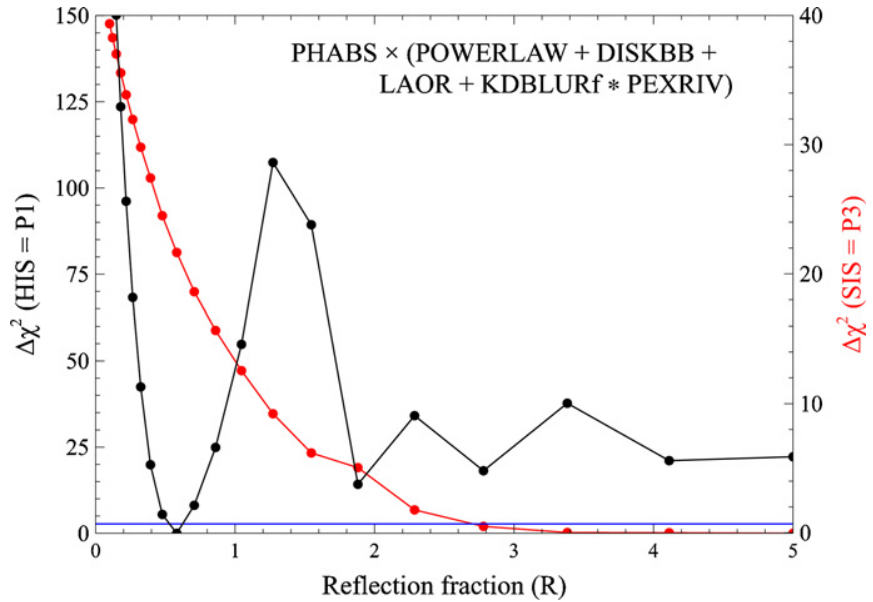

Figure 5. Goodness of fit vs. reflection fraction for the two representative spectra describing the HIS-P1 (black) and SIS-P3 (red). The spectra used refer to those highlighted in Figures 2 and 4 and the REFLIONX component inherent in the base model has been replaced with a combination of PEXRIV together with LAOR. The solid blue horizontal line shows the $90 \%$ confidence range.

(A color version of this figure is available in the online journal.) 

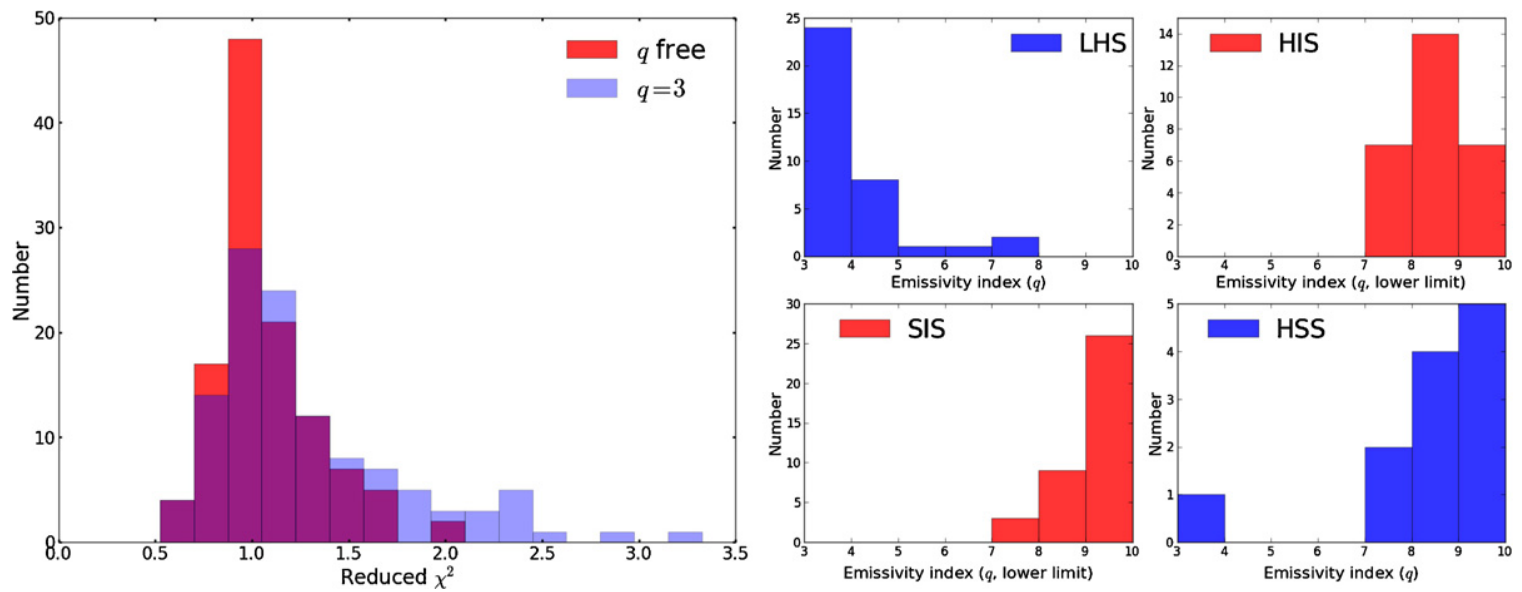

Figure 6. Left: distribution of reduced- $\chi^{2}$ for model with a Newtonian emissivity profile $(q=3)$ and a steeper values $\geqslant 3$. In both cases we see a peak at reduced$\chi^{2}=1$, however this is much clearer after allowing the emissivity to vary beyond its Newtonian value. Right: distribution of emissivity parameter (blue) and their $90 \%$ lower limit (red) for the four states highlighted in each panel. Bottom: in all cases, the emissivity index was constrained to $3 \leqslant q \leqslant 10$.

(A color version of this figure is available in the online journal.)

be constrained when the PCA data are at its highest level as this constraint does not come from energies $>25 \mathrm{keV}$. At the end of the outburst, when the PCA signal-to-noise level drops significantly, the data cannot differentiate between a Newtonian $q=3$ and a steeper value.

Following the recipe provided for Cygnus X-1 by Fabian et al. (2012a) in dealing with sources where the spin is expected to be high (as is the case for XTE J1650-500), we have repeated our fits with a double emissivity profile such that within a break radius (initially frozen at $4 r_{\mathrm{g}}$ but later allowed to vary) the emissivity is $>3$ and beyond it is frozen at 3 . The initial value of $4 r_{\mathrm{g}}$ for the break radius was chosen based upon the value for Cygnus X-1 (Fabian et al. 2012a). We find that, as long as the emissivity is not fixed at $q=3$, the quality of the fits, and distribution of reduced- $\chi^{2}$ are similar to that of a single, unbroken emissivity, and we proceed by using this single power-law emissivity profile as our standard but emphasize that the results presented here do not change if we employ a broken emissivity profile. This is very likely to be due to the comparatively low spectral resolution afforded by $R X T E$, which does not reflect the subtle changes in the reflection profile in a similar manner as XMM-Newton or Suzaku observations.

\subsection{Light Bending and General Relativity}

Hints of the expected effects of light bending, as described in the introduction, can be seen in the top four panels in Figure 3. Most important is the apparent constancy of the reflection flux in comparison with that of the direct power law early in the outburst. We investigate this further in Figure 7. The left panel shows the flux-flux relation through the whole outburst with the various spectral states shown in different colors. The top-right panel is a close up of the period covering the HIS and SIS during the first $\sim 30$ days of the outburst. ${ }^{13}$ Figure 7 is remarkably similar to Figure 3 of Rossi et al. (2005), where the authors used the flux in the iron line as a proxy for the total reflection in XTE J1650-500. We have superimposed in this figure the expectation from the light-bending model for a compact corona varying in height from $1-20 r_{\mathrm{g}}$ with a disk having an inclination of $60^{\circ}$, from Miniutti \& Fabian (2004, see their 13 Excluding the first 3 days when XTE J1650-500 was in the rising LHS (see
Figure 1).
Figure 2). In order to correctly describe the shape of the function shown graphically in Miniutti \& Fabian (2004), we used the Dexter Java application of Demleitner et al. (2001) to obtain a fourth-order polynomial fit to their curve from which we applied a linear normalization of $1.5 \times 10^{-9}$ and $4.5 \times 10^{-9}$ to their $Y$ (arbitrary Fe-line flux) and $X$-axis (arbitrary power-law flux), respectively. The model reproduces extremely well the broad shape of the relation. Finally, the bottom right panel shows this behavior when the non-extrapolated, $3-100 \mathrm{keV}$ fluxes are used instead. ${ }^{14}$ We again see that qualitatively, the behavior is the same as above.

As discussed in the introduction, the light-bending model of Miniutti et al. (2004) predicts the existence of semi-distinct regimes in this flux-flux relation. When the corona is located at a height of $\sim 10 r_{\mathrm{g}}$ the model predicts a flattening in the relation similar to that observed for the HIS (both P1 and P2). The fluxes in this HIS are clearly correlated, and a Spearman's rank correlation test gives a coefficient of $\rho=-0.799$ corresponding to a $1.7 \times 10^{-6}$ chance of a false correlation (Table 1$)$. The slope of this relation is $-0.24 \pm 0.02$ (standard error (s.d.)) and this linear fit is shown in the top-right panel of Figure 7 as a solid black line.

As the location of the corona reaches the more extreme environment within a few $r_{\mathrm{g}}$ from the black hole, the model predicts a steep, positive linear relation between the reflection and power-law flux similar to that seen in the SIS, although there is large scatter dominated by poor statistics. A Spearman's rank correlation test here gives $\rho=0.719$, with a false correlation probability of just $2.4 \times 10^{-6}$. The slope of this relationship is greater than unity, with a best-fit value of $2.7 \pm 0.1$ (shown as a further solid black line in Figure 7). Note that this is highly inconsistent with the expectation for a static Newtonian corona with intrinsically varying luminosity, where the slope should be unity across the entire flux range. It is the combination of a slope $\gg 1$ at low power-law flux together with a near-flattening at higher fluxes that provides evidence for relativistic effects in this case.

It has been suggested (e.g., Ballantyne et al. 2003, 2011) that changes in the ionization of the inner regions of the accretion disk can give rise to changes the reflection spectrum that can

\footnotetext{
${ }^{14}$ In this case the normalizations were $1.5 \times 10^{-9}$ and $4.5 \times 10^{-9}$ for the
} Fe-line and power-law flux, respectively). 

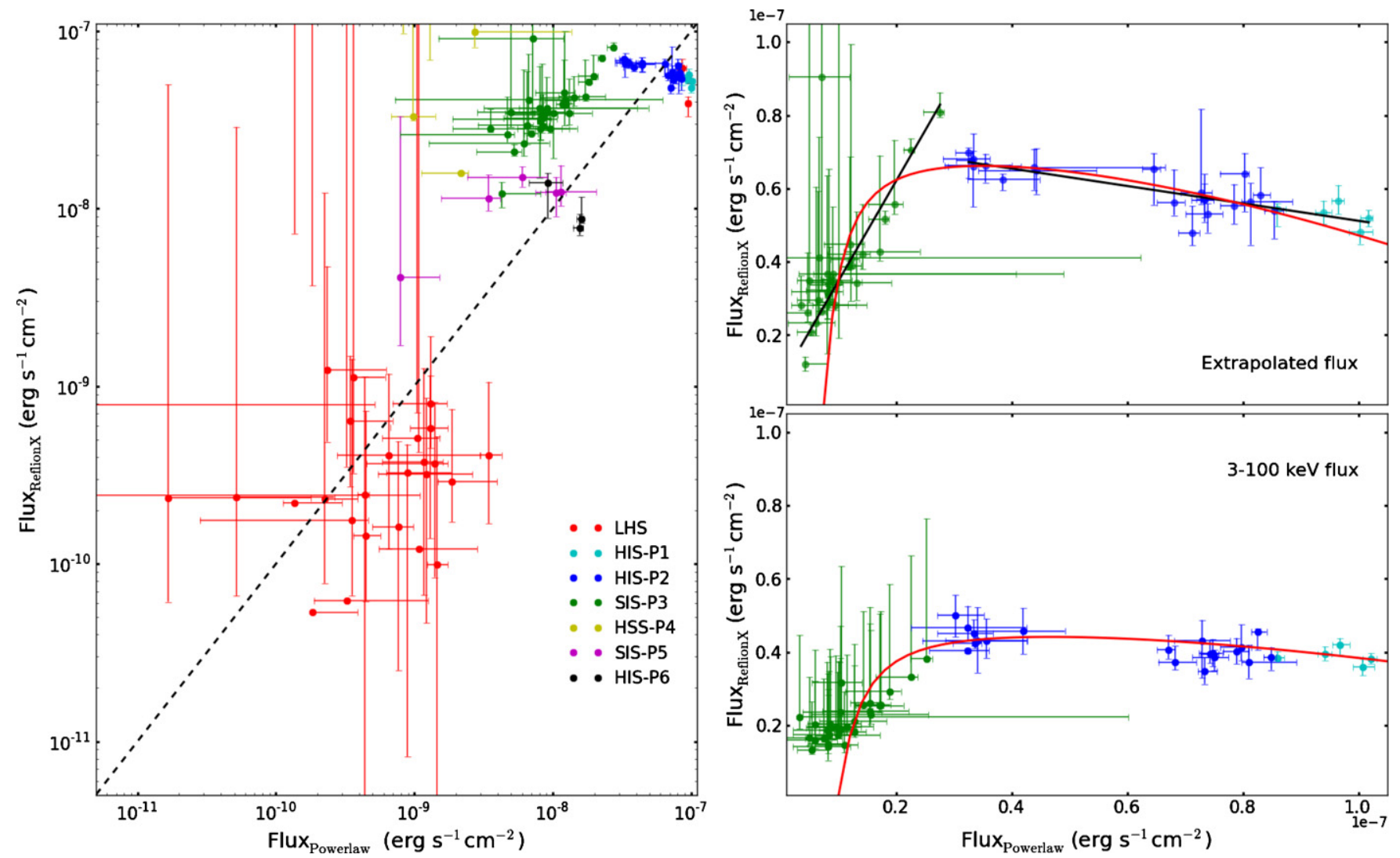

Figure 7. Left: flux-flux relation during the outburst. The dashed line shows the one-to-one relation. Top right: close up of the HIS (blue and cyan) and SIS (green). The solid black lines show the best linear fit for each state having a slope of $2.7 \pm 0.1$ and $-0.24 \pm 0.02$ in the SIS and HIS, respectively. The solid red curve shows the expected flux-flux relation under the light-bending model of Miniutti \& Fabian (2004, see their Figure 2) for a system with $60^{\circ}$ inclination, somewhat similar to XTE J1650-500, with a corona varying in height from 1 to $20 r_{\mathrm{g}}$. Note that this is not a fit and has been rescaled from the original (see Section 4.3). Bottom right: similar but for fluxes between 3 and $100 \mathrm{keV}$.

(A color version of this figure is available in the online journal.)

Table 1

Summary of Spearman's Rank Correlations and Partial Correlation Tests on a Combination of Various Model Parameters

\begin{tabular}{|c|c|c|c|c|c|c|c|c|c|}
\hline \multirow[b]{3}{*}{ Parameter 1} & \multirow[b]{3}{*}{ Parameter 2} & \multicolumn{4}{|c|}{ HIS (P1 + P2) } & \multicolumn{4}{|c|}{ SIS (P3) } \\
\hline & & \multicolumn{2}{|c|}{$\begin{array}{l}\text { Spearman's } \\
\text { Rank-order }\end{array}$} & \multicolumn{2}{|c|}{$\begin{array}{c}\text { Partial } \\
\text { Correlation }\end{array}$} & \multicolumn{2}{|c|}{$\begin{array}{l}\text { Spearman's } \\
\text { Rank-order }\end{array}$} & \multicolumn{2}{|c|}{$\begin{array}{c}\text { Partial } \\
\text { Correlation }\end{array}$} \\
\hline & & $\rho$ & $p$-value & $\rho$ & $p$-value & $\rho$ & $p$-value & $\rho$ & $p$-value \\
\hline$\xi$ & $F_{\text {powerlaw }}$ & -0.953 & $2.0 \times 10^{-13}$ & -0.473 & 0.014 & 0.319 & 0.071 & 0.092 & 0.621 \\
\hline$\xi$ & $F_{\text {disk }}$ & 0.956 & $9.3 \times 10^{-14}$ & 0.515 & 0.006 & 0.006 & 0.972 & 0.082 & 0.656 \\
\hline$F_{\text {disk }}$ & $F_{\text {powerlaw }}$ & -0.955 & $1.1 \times 10^{-13}$ & -0.427 & 0.0031 & -0.310 & 0.079 & -0.334 & 0.056 \\
\hline$\xi$ & $F_{\text {reflionX }}$ & 0.742 & $2.2 \times 10^{-5}$ & -0.140 & 0.516 & 0.382 & 0.028 & 0.218 & 0.230 \\
\hline$F_{\text {disk }}$ & $F_{\text {reflionX }}$ & 0.766 & $7.8 \times 10^{-6}$ & 0.088 & 0.685 & -0.116 & 0.520 & 0.140 & 0.446 \\
\hline$F_{\text {powerlaw }}$ & $F_{\text {reflionX }}$ & -0.799 & $1.7 \times 10^{-6}$ & -0.373 & 0.066 & 0.719 & $2.4 \times 10^{-6}$ & 0.683 & $4.6 \times 10^{-7}$ \\
\hline
\end{tabular}

Notes. Spearman's rank correlations and partial correlation test were made for combinations of the reflection, power-law, and disk fluxes as well as the ionization parameter. The partial correlation test measures the degree of associations between the two parameters listed on the first two columns while controlling for the remaining two parameters. The Spearman's coefficient $\rho$ is a measure of the degree of correlation with +1 or -1 indicating a perfect monotone function and 0 a lack of correlation.

mimic somewhat this flat behavior. In order to robustly assess the strength of the correlations seen in Figure 7 in both the HIS and SIS, we have performed a number of correlation tests which are summarized in Table 1. We performed, for both HIS and SIS, Spearman rank-order tests for a combination of four parameters (power-law, reflection, and disk fluxes as well as the disk ionization) as well as partial correlation tests (PCTs) for two parameters while controlling for the third and fourth variable. The PCT is of particular importance for our purpose as it removes any potential association of the ionization parameter (or any other potential source of unwanted correlation) in the flux-flux relations shown in Figure 7.

From the Spearman rank-order tests performed in the HIS (Table 1), it would initially appear that all four variables are strongly correlated with one another in some way, as all combinations display $|\rho| \gtrsim 0.7$. However, after performing the PCT for all combinations we see that for two of the previous strong correlations $\left(\xi-F_{\text {reflionX }}\right.$ and $\left.F_{\text {disk }}-F_{\text {reflionX }}\right)$ were in fact driven by the mutual dependence of these parameters on $F_{\text {powerlaw. }}$. These tests clearly indicate that the reflection flux in 


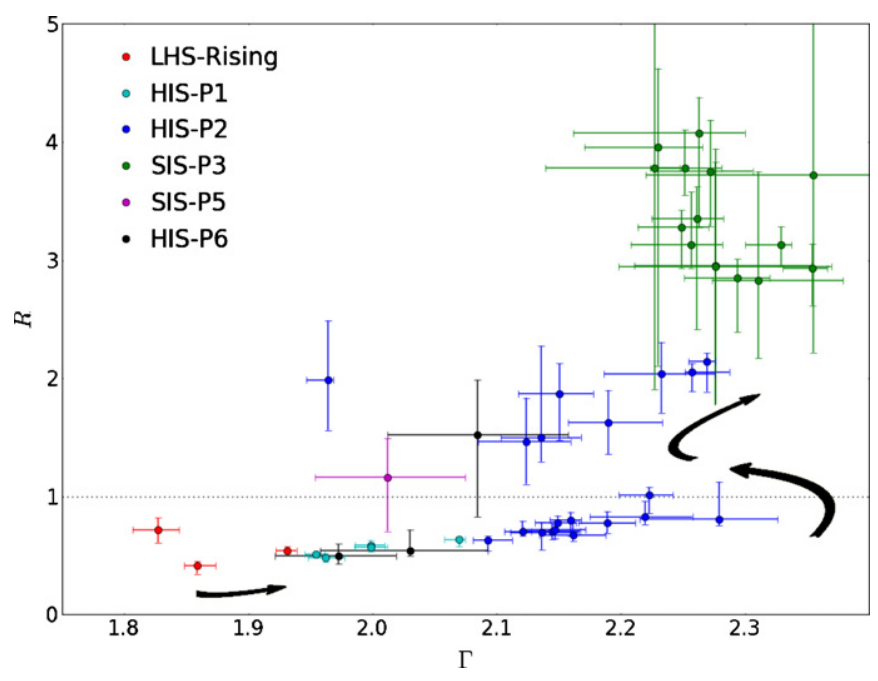

Figure 8. Well-known $R-\gamma$ relation appears present in the rising LHS and during most of the HIS. However, just prior to the transition to the SIS and thereafter, this relation does not hold. We discuss a potential explanation for this behavior in Section 4.4. Only data with errors less than $50 \%$ their value are used in this figure. The black arrows show approximately the evolution of the system in time.

(A color version of this figure is available in the online journal.)

both states is better correlated with the power-law flux than with ionization and the slope of the correlations (mildly negative in the HIS and strongly positive in the SIS) are highly indicative of gravitational light bending in the GR regime.

The behavior seen here is consistent with a drop in the height of the corona during the hard-intermediate phase (P1 and P2) followed by intrinsic variations in its luminosity by a factor of a few during the SIS (P3). Following the disk-dominated soft-state (P4), the height of the corona increases again (P5 and P6) and the outburst finishes with the intrinsic power dropping as the source goes back into the LHS.

\section{4. $R-\Gamma$ Relation}

A strong correlation has been shown to exist between the amplitude of the reflected component $(R)$ and the photon index $(\Gamma)$ of the Comptonized spectrum in XRBs in the hard state (e.g., Ueda et al. 1994). This $R-\Gamma$ relation has since been robustly tested by a number of authors (e.g., Gilfanov et al. 1999; Zdziarski et al. 1999, 2003; Nowak et al. 2002; Ibragimov et al. 2005) and it is now thought to also apply to Seyfert galaxies and radio galaxies (e.g., Zdziarski et al. 1999), further cementing the similarities in the coronal properties at all scales. If this relation indeed turns out to be real (and evidence attests to this; but see Molina et al. 2009) then this is could be telling us about the feedback between the hot corona and cold gas in an accretion disk.

We briefly investigate this relationship for XTE J1650-500 in Figure 8. For clarity, we only consider data with a fractional uncertainty of less than $50 \%$. The black arrows approximately show the evolution of the system in time. Although as a whole the data do not strongly support the presence of a relationship between $R$ and $\Gamma$, when the states are roughly separated (different colors) it does appear that early in the outburst through to the last few days of the HIS the relation seems to hold. It is clear, at least, that the rising LHS and the HIS populate different regions in the figure to the SIS.

The potential presence of this relation early in the outburst suggests a feedback process between the soft photons in the disk and the corona. There are a number of theoretical interpretations for the presence of this correlation (Poutanen et al. 1997; Gilfanov et al. 1999, 2000; Beloborodov 1999b; Malzac et al. 2001) with the two leading contenders often described as the disk-truncation and dynamic corona model (see Done 2002; Beloborodov 1999a, for a detailed study of these models). To summarize, in the former, an increase in the reflection fraction is caused by the accretion disk penetrating deeper into a central hot corona, thus receiving more illuminating hard photons. The presence of the disk in return offers more soft photons consequently cooling the plasma. For a purely thermal distribution of electrons, the greater the number of soft seed photons, the softer the power-law spectra. The latter model by Beloborodov (1999b) invokes bulk motion of the corona above the accretion disk. If the corona is outflowing with mildly relativistic velocities, this would reduce the amount of hard photons hitting the disk, which in turn reduces $R$ and subsequently softens $\Gamma$ as few reprocessed soft photons reach the outflow. Recent evidence for the coronal plasma ejection model has come from a strong positive correlation between reflected $\mathrm{X}$-ray flux and radio flux in the black hole binary Cygnus X-1 (Miller et al. 2012).

We will show in Section 4.6 that all evidence points toward the disk radius remaining stable during the HIS to SIS transition (see Figures 11 and 12). This constancy effectively rules out the "disk-truncation" explanation for the $R-\Gamma$ correlation. Instead, one may hypothesize whether the "outflowing corona" and light bending can be combined to explain the behavior so far detailed for XTE J1650-500. In the previous section we showed that the flux-flux behavior (Figure 7) could be explained if early in the outburst (during the HIS) the corona was located relatively far $\left(\sim 10 r_{\mathrm{g}}\right)$ from the black hole and thus behaved according to Regime 2 of Miniutti \& Fabian (2004; see also Section 1.2). As the system evolved, the corona collapsed to a few $r_{\mathrm{g}}$ and began to experience a higher level of light bending toward the disk (Regime 1). In this scenario, a potential gradient in the outflow velocity of the corona as a function of height could also explain the behavior seen in Figure 8. That is, as the corona collapses from a large height (large outflow velocity, low $R$ and hard $\Gamma$ ) the outflow velocity decreases ( $R$ increases and $\Gamma$ softens) until it becomes effectively static and the system transitions into the SIS. We will expand upon this possible scenario in what follows and summarize our ideas in Section 5.

\subsection{QPOs and Spectral States: A Collapsing Corona}

Throughout this work, we followed the selection made by Homan et al. (2003) which roughly separates the outburst into six periods coinciding with significant changes in both the HID (Figure 2) as well as in the shape of their power spectra. ${ }^{15}$ As highlighted in the Introduction (Section 1.3), those authors demonstrate the presence of high-frequency (HF) variability in XTE J1650-500 together with an HF QPO which was shown to evolve in both frequency and coherence during the outburst. The highest frequency which was reliably measured was at $\sim 250 \mathrm{~Hz}$ in the SIS, with the frequency being much lower $(\sim 50 \mathrm{~Hz})$ at the onset of the outburst.

In Figure 9 (top left), we show the presence of a strong (Pearson's $r=0.997)$ positive relation between the reflection fraction and quality factor of the QPO $(Q-R$ relation $)$. In order

\footnotetext{
15 In keeping with that work, the HIS is divided into two periods ( $\mathrm{P} 1$ and $\mathrm{P} 2$ ). Here, we also add two extra periods which we have denoted as the LHS rising and falling.
} 

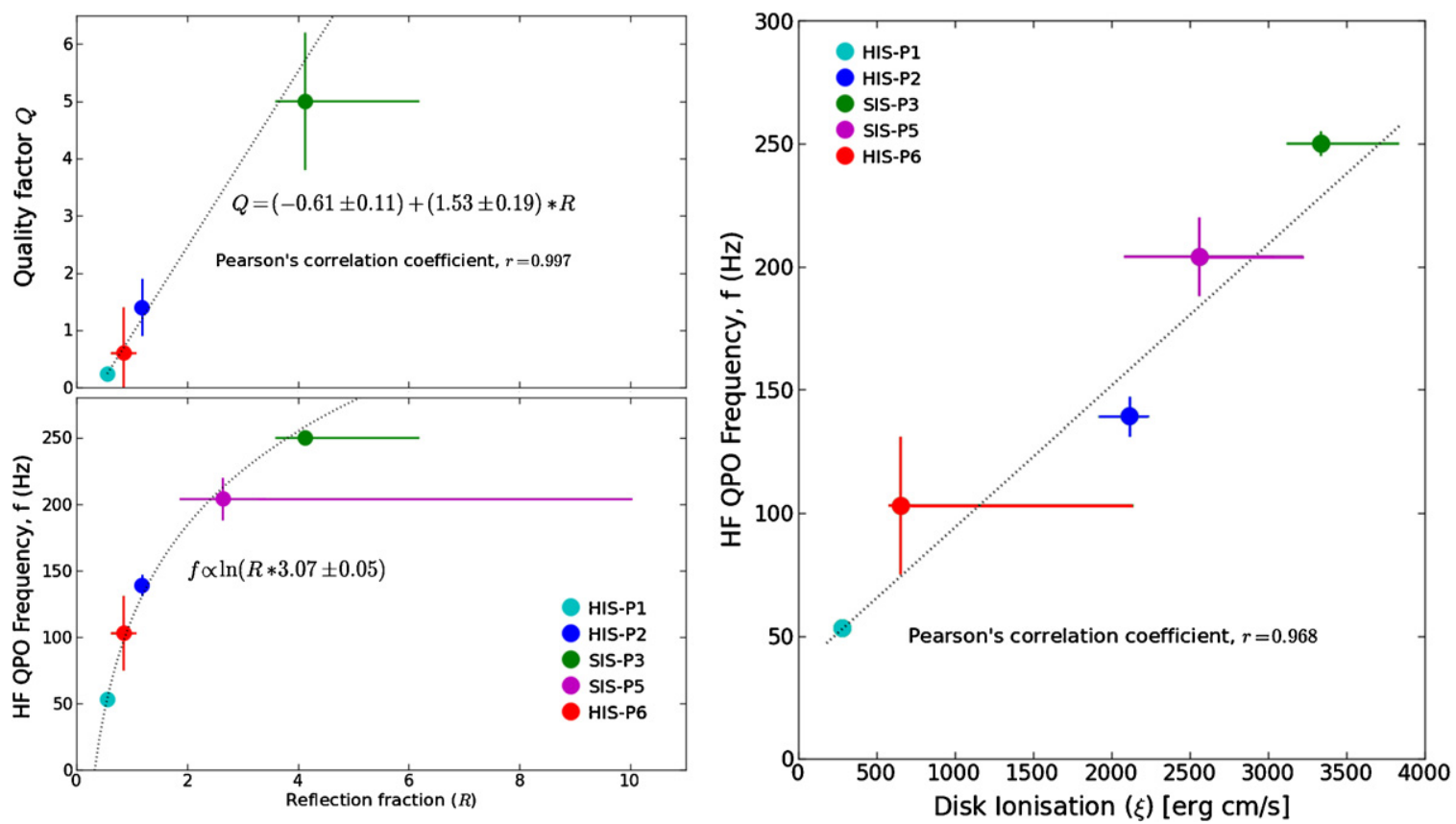

Figure 9. Top left: HF QPO coherence as a function of reflection fraction. The relation is well described by a linear function similar to that shown in the figure. Bottom left: QPO frequency as a function of reflection fraction. The dashed line shows a relation, $f(\mathrm{~Hz})=(102 \pm 2) \times \ln [R \times(3.07 \pm 0.05)]$, that fits these data. Right: QPO frequency as a function of disk surface ionization. These figures show a clear link between the coherence/frequency of the QPO and the reflection fraction or level of disk ionization, which we subsequently interpret as being linked to the size/position of the corona (see Section 4.5).

(A color version of this figure is available in the online journal.)

to create this figure, we have averaged the values of $R$ shown in Figure 3 for each of the periods in question and used the values for the coherence provided by Homan et al. (2003; Table 1). The bottom left panel shows the frequency of the HF QPO as a function of $R(f-R$ relation). We also show in Figure 9 (right), the QPO frequency as a function of disk (surface) ionization parameter.

QPOs are notoriously difficult to explain and it is not our purpose to provide a quantitative description of this phenomenon. However, it is worth stressing that most models (e.g., Nowak et al. 1997; Cui et al. 1998; Psaltis et al. 1999; Stella et al. 1999; McKinney et al. 2012) strongly link the origin of QPOs with orbits and/or resonances in the inner accretion disk close to the black hole. Current models cannot fully explain, in a physical manner, the range in coherence observed in various systems nor the manner in which the frequencies change with states.

To explain the range in coherence observed in accreting neutron stars, Barret et al. $(2006,2007)$ devised a toy model in which the changes in $Q$ are driven by changes in the scale height of the disk. A small-scale height gives rise to high coherence and vice versa. Expanding on this idea, it appears that at least for XTE J1650-500, it is physical changes in the radius/size of the corona that give rise to changes in both quality factor $Q$ and QPO frequency. To illustrate this hypothesis, consider Figure 9 (bottom) together with our interpretation of the behavior displayed in Figure 7 (Section 4.3). Early in the outburst the frequency of the HF QPO appeared at $\sim 55 \mathrm{~Hz}$. The Keplerian frequency at a given radius is $f(\mathrm{~Hz}) \approx$ $3.2 \times 10^{4} M^{-1} r^{-3 / 2}$, where $M$ and $r$ are in units of solar mass mass and gravitational radius, respectively. Hence, during the brief P1 period, the HF QPO frequency is close to the orbital frequency at $\sim 28 r_{\mathrm{g}}$ assuming a $4 M_{\odot}$ black hole and potentially moves to $\sim 15 r_{\mathrm{g}}$ in the second half of the HIS. As the outburst continues and the corona continues to collapse, it is plausible that the frequency continues to increase (corresponding to $\sim 10 r_{\mathrm{g}}$ in the SIS), eventually approaching a value that should be consistent with the Keplerian frequency at the ISCO. The continued decrease in the size of the corona gives rise to the increase in coherence. In this scenario, the frequency of the QPO should relate to the size of the corona and thus would naturally increase as the corona collapses. The relationship between the QPO frequency and the surface ionization parameter could be suggestive of an intrinsic relationship between the irradiation of the disk and its magnetic field properties, the latter of which has recently been proposed as a possible means to produce (low-frequency) QPOs (see, e.g., O’Neill et al. 2011; Oishi \& Mac Low 2011). This possibility will be addressed in forthcoming work.

Finally, the relation between the coherence of the HF QPOs and the reflection fraction also leads to the interesting prediction that HF QPOs should only be observed when $R \gtrsim 0.4$ when the coherence is $Q>0$. This result is consistent with observations, with no HF QPO ever having been found in the LHS, where $R \lesssim 1$

\subsection{Radio (Jet) Emission and Reflection Fraction}

Corbel et al. (2004) presented a comprehensive analyses of the radio emission observed during the outburst of XTE J1650-500. In that work, the authors suggest that the transition between the HIS and SIS ${ }^{16}$ is associated with a massive radio ejection event. The observations in the LHS were found to be consistent with the presence of a steady, compact jet, as is often seen in the LHS of black hole binaries (see, e.g., Fender 2001; Fender et al. 2009, and references therein). This potential ejection event during the HIS-SIS transition coincides with the time where we

\footnotetext{
${ }^{16}$ Referred to as the intermediate and steep power-law states, respectively, in Corbel et al. (2004).
} 


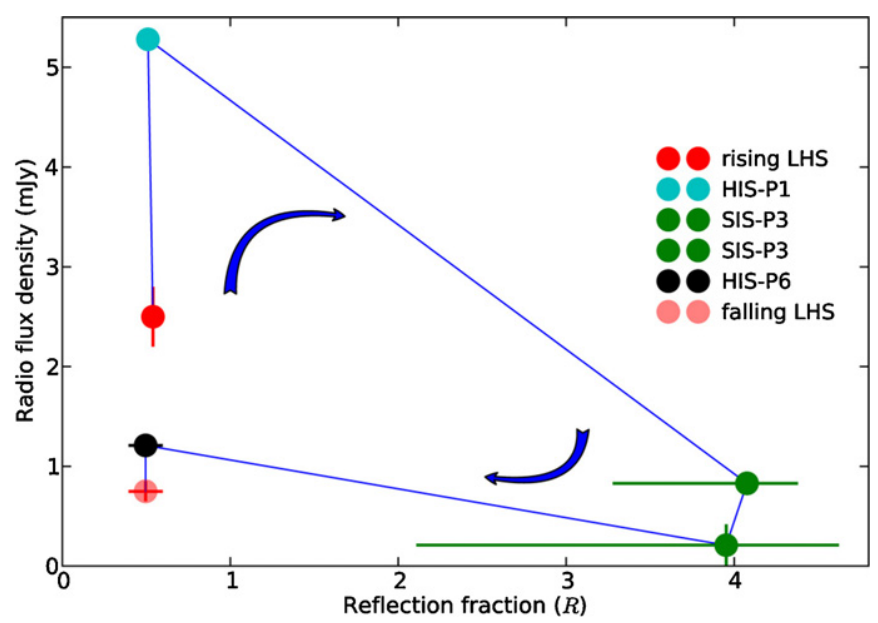

Figure 10. Radio-flux density as a function of reflection fraction. The arrows show the direction of the outburst. There appears to be two distinct branches where the reflection fraction is either constant at $R \sim 0.5$ or at $R \sim 4$. These two branches correspond to the LHS/HIS and SIS, respectively. Radio data obtained from Corbel et al. (2004).

(A color version of this figure is available in the online journal.)

see a sharp jump in the reflection fraction. In Figure 10 we show the radio-flux density ${ }^{17}$ versus the reflection fraction calculated herein. At the time of the steady compact jet (in the LHS and HIS), the radio-flux density increased by a factor of $\sim 5$ with no statistically significant change in $R$. However, immediately following the radio ejection in the SIS, the reflection fraction increases dramatically resulting in a bi-modality in the radioflux density-reflection fraction plane. This suggests an intimate link between the jet ejection site and the collapsing corona. Indeed, Beloborodov (1999b) predicts a link between radio jets and reflected flux, which was also seen in Cygnus X-1 (Miller et al. 2012).

At later stages in the outburst (HIS-P6, falling LHS), the measured radio-flux density is likely a dominated by emission from the zone where the ejected plasma interacts with the

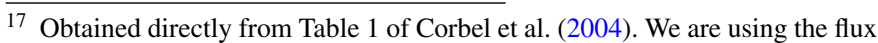
densities at $4800 \mathrm{MHz}$ for all observations, except for the rising LHS where this was not available. In this case, we proceeded by averaging the values presented for 1384 and $2496 \mathrm{MHz}$. ambient ISM surrounding the binary system. Unfortunately, due to the low spatial resolution of the radio observations, this emission is not resolved from that due to any reformation of the steady jet close to the black hole.

\subsection{Disk (Inner) Radius and State Transition}

As has been discussed throughout this paper, a popular explanation for state transitions is a radial variation in the extent of the accretion disk. This model has been highly successful in part due to its flexibility and ease in which it can explain the "weak" reflection fraction $(R<1)$ often found in the LHS. However, over the past few years it has consistently been shown that in the luminous phases of the LHS - at least above $\sim 1 \times 10^{-3} L_{\text {Edd }}{ }^{18}$ - the disk does not appear to be truncated away from the radius of the ISCO (Miller et al. 2006a, 2006b, 2008b; Reis et al. 2009c, 2010; Reynolds \& Miller 2011; Walton et al. 2012). Figure 11 (left) shows that the present work can statistically rule out a disk being truncated further than $\sim 3 r_{\text {g }}$ even in the LHS. During the brighter, ISs, we constrain this radius to $\sim 1.65 r_{\mathrm{g}}$. This adds support to the idea that the inner disk radius remains roughly constant throughout the LHS-HIS-SIS state transitions in black hole binaries.

Where we have not been able to constrain the radius, this has largely been due to the data quality (the falling phase of the LHS is inherently less luminous) as well as the fact that reflection is intrinsically weaker in the LHS. The strongest reflection features are expected in the ISs where the disk receives a larger fraction of the hard X-ray emission (see, e.g., Hiemstra et al. 2011). Note also that despite the comparatively low spectral resolution afforded by the RXTE/PCA (18\% FWHM energy resolution at $5.9 \mathrm{keV}$ ) Wilms et al. (2006) showed that this instrument can indeed resolve line widths down to a least $\sigma \sim 0.3 \mathrm{keV}$. Higher resolution observations of XTE J1650-500 early in the outburst showed that, when modeled with a "Gaussian",

\footnotetext{
18 Contrast this with the broadband analyses of GX 339-4 presented by Tomsick et al. (2009), where the authors find clear evidence for the recession of the accretion disk beginning only at Eddington luminosities below $\sim \times 10^{-3} L_{\text {Edd }}$.
}
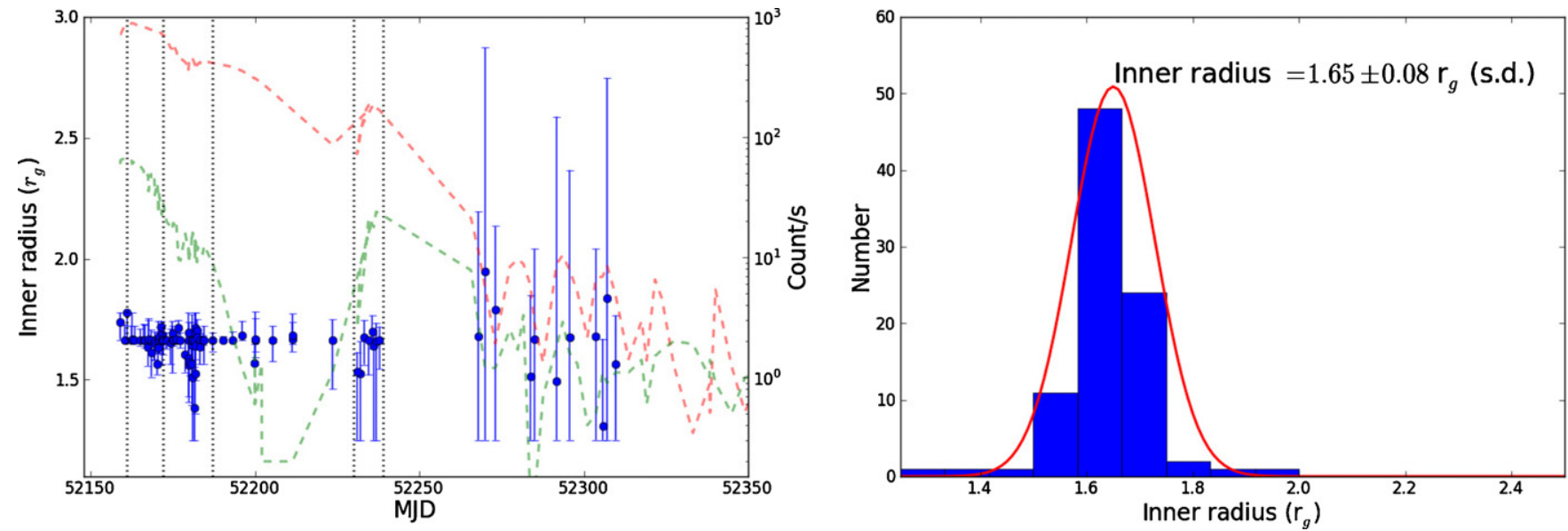

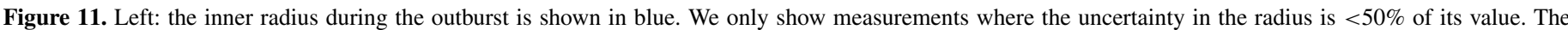

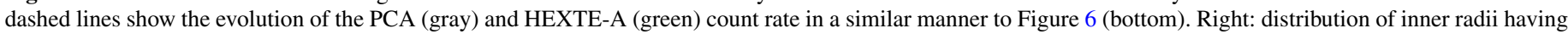
errors $\leqslant 50 \%$ its value. In red we show the standard normal distribution with a mean radius of $1.65 r_{\mathrm{g}}$ and standard deviation of $0.08 r_{\mathrm{g}}$.

(A color version of this figure is available in the online journal.) 

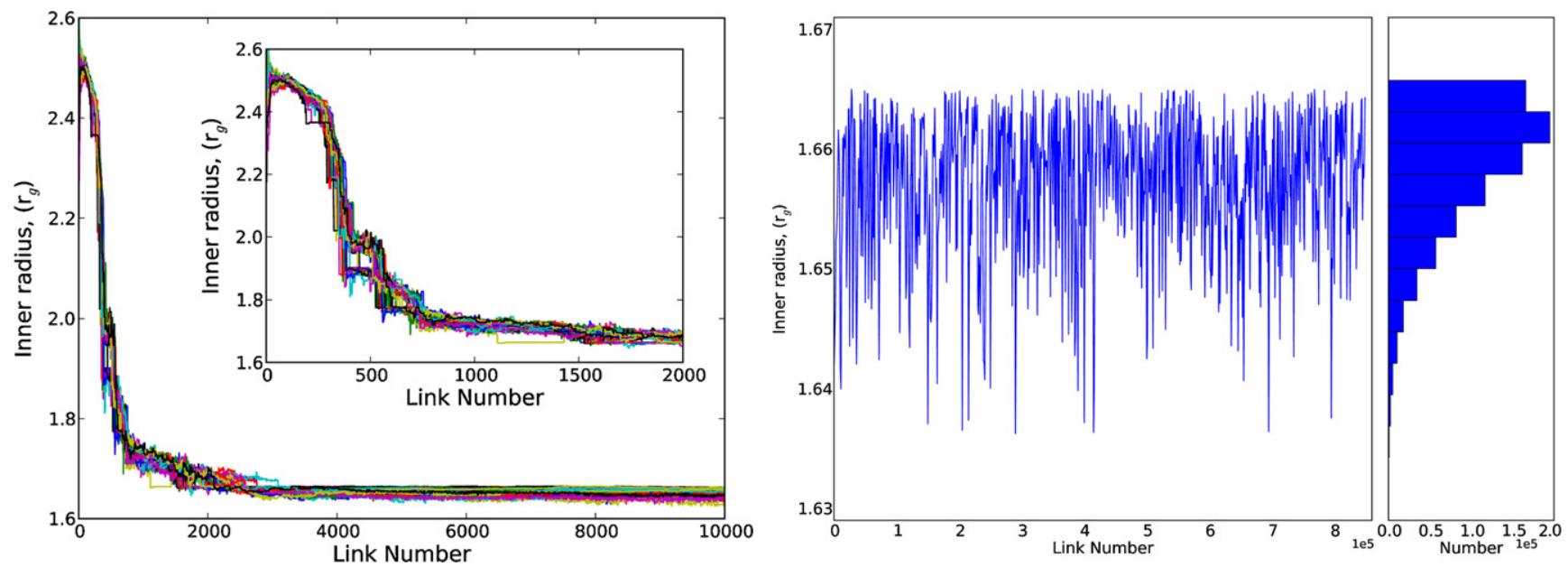

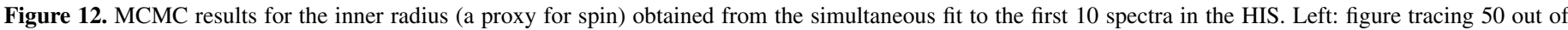

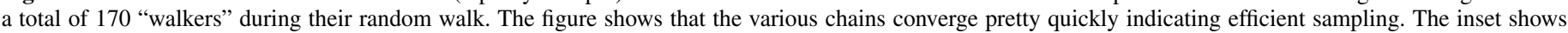

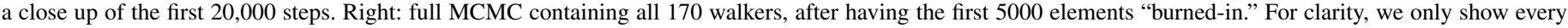
1000th element of the chain.

(A color version of this figure is available in the online journal.)

the $\mathrm{Fe}-\mathrm{K} \alpha$ emission line is consistent with having a width of $\sigma \sim 1.1 \mathrm{keV}$ (Table 4 in Walton et al. 2012). ${ }^{19}$

Assuming that the stable radius shown in Figure 11 for the two ISs is indeed the radius of the ISCO, we find, using the relationship between ISCO and black hole spin of Bardeen et al. (1972), a dimensionless spin parameter of $0.977_{-0.007}^{+0.006}$ consistent with the value found in detailed analyses of single, high-quality data obtained with XMM-Newton $(0.84 \leqslant a \leqslant 0.98$; Walton et al. 2012) or BeppoSAX ( $a \gtrsim 0.93$; Miniutti et al. 2004 ${ }^{20}$ ).

As a test of the robustness of this result, we have performed a joint fit to the first 10 observations in the HIS. We used the same base model as before with each individual observation having their own set of parameters-disk emissivity index, temperature, normalization and parameter, power-law index, as well as the normalization of the power law and reflection component. However, this time we forced the inner radius in the various observations to be a global parameter thus assuming a constant value.

This simultaneous fit contains a total of 81 free parameters ${ }^{21}$ and with this comes a high chance of mistaking a local minima in $\chi^{2}$ space for the global best fit. In order to address these limitations, we proceeded by minimizing the fit using standard $\chi^{2}$ fitting techniques within XSPEC until a reasonable fit was produced $\left(\chi^{2} / v<2\right)$ at which point we halted the minimization ${ }^{22}$ and proceeded with Monte Carlo Markov Chain (MCMC) analysis. We employed the MCMC procedure described in Foreman-Mackey et al. (2012; code found at http://danfm.ca/emcee/) and implemented in the XSPEC spectral fitting package by Jeremy Sanders (XSPEC implementation described in https://github.com/jeremysanders/xspec_emcee). MCMC techniques have been successfully used to address similar problems in constraining the black hole spin of NGC 3783 (Reynolds et al. 2012) as well as in modeling the kinematics of the microquasar XTE J1550-564 (Steiner \& McClintock 2012).

\footnotetext{
19 The original analyses of this XMM-Newton data set performed by Miller et al. (2002) included an extra smeared edge component at $\sim 6.8 \mathrm{keV}$ which resulted in the "Gaussian" having a width of only $\sim 250 \mathrm{eV}$.

20 Spin converted from the lower limit on the inner radius of $\sim 2.1 r_{\mathrm{g}}$.

21 The sheer number of free parameters and computational time required to do $\chi^{2}$ fitting as well as the MCMC analyses described in what follows drove the need to constrain this analyses to only 10 observations as opposed to all 116 .

22 The actual quality of the fit at this time was $\chi^{2} / v=2059.7 / 1149$.
}

We added a $5 \%$ random perturbation to all the parameters in the fit described above, and increased the value of the inner radius from the starting value of $\sim 1.6 r_{\mathrm{g}}$ to $2.5 r_{\mathrm{g}}$ in order to guarantee that the chain could freely converge to the global minimum. We used a total of 170 "walkers," each iterated ("walking") 10,000 times. Figure 12 (left) shows the evolution of the walk in inner radius for 50 randomly selected walkers. It is clear that the walkers converge to the same value efficiently. Nonetheless, in order to be conservative we have ignored ("burned-in") the first 5000 elements of each chain and show on the right the full MCMC chain for the radius which is clearly well behaved with a peak distribution at $1.66 \pm 0.01 r_{\mathrm{g}}$ (s.d.), in excellent agreement with the results in Figure 11.

\section{SUMMARY}

In this work, we try to take a broad systematic approach to not only the data reduction but also in the manner in which the spectra are fit during all spectral states observed during the outburst. We have presented a number of empirical results inferred from our a priori assumption that the observed spectra and their evolution is a consequence of variation in three separates emission components-the power-law continuum, thermal disk, and reprocessed reflection. Although we have tried to convey a fully consistent, albeit qualitative picture of the spectral evolution of XTE J1650-500, it is very difficult to obtain unique interpretation of the results and nearly impossible to generalize this to all systems. In a forthcoming publication, we will apply similar techniques to a larger set of objects at which point we hope to be able to make a stronger statement regarding the global population of stellar-mass black hole binaries and possibly AGNs.

For now, we summarize the main results of the presented spectral analyses as follows.

1. The outburst is well characterized by a model consisting of a hard X-ray continuum, a thermal disk component, and reprocessed emission (reflection).

2. The emissivity profile of the disk is not well characterized by a simple Newtonian approximation and, where this value can be constrained early in the outburst, it is steeper than the Newtonian value $q=3$. 
Table 2

Parameters of Interest during the Outburst of XTE J1650-500

\begin{tabular}{|c|c|c|c|c|c|c|c|c|c|c|}
\hline \multirow[t]{2}{*}{ MJD } & \multicolumn{4}{|c|}{ Flux $\left(\times 10^{-10} \mathrm{erg} \mathrm{cm}^{-2} \mathrm{~s}^{-1}\right)$} & \multirow{2}{*}{$\begin{array}{c}\text { Disk Temperature } \\
(\mathrm{keV})\end{array}$} & \multirow{2}{*}{$\begin{array}{c}\text { Ionization } \\
\xi\left(\mathrm{erg} \mathrm{cm} \mathrm{s}^{-1}\right)\end{array}$} & \multirow[t]{2}{*}{$\Gamma$} & \multirow{2}{*}{$\begin{array}{l}\text { Reflection Fraction } \\
\qquad(R)\end{array}$} & \multirow{2}{*}{$\begin{array}{l}\text { Emissivity } \\
\text { Index }(q)\end{array}$} & \multirow{2}{*}{$\begin{array}{c}\text { Disk } \\
\text { Inner Radius (rg) }\end{array}$} \\
\hline & Total $^{\dagger}$ & Power Law ${ }^{\dagger}$ & Reflection ${ }^{\ddagger}$ & $\operatorname{Disk}^{\dagger}$ & & & & & & \\
\hline 52158.99 & $1246_{-46}^{*}$ & $954_{-42}^{+36}$ & $393_{-63}^{+34}$ & $*$ & $0.128_{-0.028}^{+1.372}$ & $235_{-9}^{+16}$ & $1.86_{-0.01}^{+0.02}$ & $0.412_{-0.069}^{+0.039}$ & $10.0_{-2.4}^{+0.0}$ & $1.74_{-0.07}^{+0.04}$ \\
\hline 52159.14 & $1348_{-86}^{+114}$ & $861_{-63}^{+60}$ & $616_{-81}^{+77}$ & $21_{-12}^{+15}$ & $1.050_{-0.383}^{+0.126}$ & $223_{-46}^{+34}$ & $1.83_{-0.02}^{+0.02}$ & $0.715_{-0.108}^{+0.103}$ & $10.0_{-7.0}^{+0.0}$ & $19.99_{-18.74}^{+24.46}$ \\
\hline 52160.39 & $1402_{-24}^{+44}$ & $978_{-12}^{+12}$ & $528_{-23}^{+33}$ & $26_{-12}^{+53}$ & $0.523_{-0.137}^{+0.126}$ & $239_{-5}^{+5}$ & $1.93_{-0.01}^{+0.01}$ & $0.540_{-0.024}^{+0.035}$ & $7.5_{-0.3}^{+0.7}$ & $1.67_{-0.00}^{+0.06}$ \\
\hline 52161.11 & $1432_{-23}^{+81}$ & $1018_{-14}^{+8}$ & $519_{-23}^{+22}$ & $33_{-12}^{+7}$ & $0.451_{-0.114}^{+0.158}$ & $242_{-5}^{+9}$ & $1.95_{-0.01}^{+0.01}$ & $0.510_{-0.024}^{+0.022}$ & $9.3_{-1.7}^{+0.5}$ & $1.78_{-0.11}^{+0.00}$ \\
\hline 52162.45 & $1424_{-33}^{+42}$ & $966_{-12}^{+9}$ & $566_{-36}^{+43}$ & $37_{-13}^{+30}$ & $0.507_{-0.094}^{+0.093}$ & $249_{-4}^{+6}$ & $2.00_{-0.01}^{+0.01}$ & $0.586_{-0.038}^{+0.045}$ & $7.3_{-0.4}^{+0.4}$ & $1.67_{-0.00}^{+0.06}$ \\
\hline 52162.59 & $1398_{-30}^{+35}$ & $1003_{-20}^{+22}$ & $481_{-33}^{+35}$ & $33_{-10}^{+21}$ & $0.622_{-0.113}^{+0.114}$ & $304_{-24}^{+29}$ & $1.96_{-0.01}^{+0.02}$ & $0.480_{-0.034}^{+0.036}$ & $8.5_{-0.6}^{+1.5}$ & $1.67_{-0.00}^{+0.11}$ \\
\hline 52163.44 & $1365_{-22}^{+25}$ & $940_{-14}^{+13}$ & $534_{-30}^{+33}$ & $29_{-5}^{+7}$ & $0.725_{-0.097}^{+0.083}$ & $285_{-12}^{+12}$ & $2.00_{-0.01}^{+0.01}$ & $0.568_{-0.033}^{+0.036}$ & $7.7_{-0.4}^{+0.8}$ & $1.67_{-0.00}^{+0.06}$ \\
\hline 52165.16 & $1290_{-13}^{+21}$ & $859_{-9}^{+15}$ & $543_{-48}^{+16}$ & $47_{-9}^{+6}$ & $0.640_{-0.024}^{+0.067}$ & $332_{-0}^{+9668}$ & $2.07_{-0.01}^{+0.00}$ & $0.632_{-0.056}^{+0.022}$ & $8.1_{-0.7}^{+0.6}$ & $1.66_{-0.02}^{+0.04}$ \\
\hline 52166.09 & $1351_{-50}^{+20}$ & $831_{-13}^{+17}$ & $582_{-30}^{+75}$ & $72_{-10}^{+4}$ & $0.633_{-0.045}^{+0.045}$ & $415_{-46}^{+12}$ & $2.12_{-0.01}^{+0.01}$ & $0.700_{-0.038}^{+0.091}$ & $8.0_{-0.4}^{+0.9}$ & $1.67_{-0.01}^{+0.07}$ \\
\hline 52166.56 & $1300_{-39}^{+33}$ & $854_{-18}^{+15}$ & $538_{-75}^{+27}$ & $69_{-15}^{+21}$ & $0.597_{-0.055}^{+0.0073}$ & $383_{-40}^{+14}$ & $2.09_{-0.01}^{+0.02}$ & $0.630_{-0.089}^{+0.033}$ & $8.3_{-1.0}^{+1.4}$ & $1.67_{-0.03}^{+0.06}$ \\
\hline 52167.49 & $1318_{-43}^{+46}$ & $785_{-24}^{+17}$ & $553_{-49}^{+59}$ & $130_{-15}^{+17}$ & $0.607_{-0.031}^{+0.031}$ & $573_{-23}^{+35}$ & $2.14_{-0.02}^{+0.02}$ & $0.704_{-0.066}^{+0.076}$ & $7.8_{-0.7}^{+0.6}$ & $1.64_{-0.08}^{+0.03}$ \\
\hline 52167.56 & $1297_{-52}^{+65}$ & $803_{-43}^{+7}$ & $640_{-103}^{+56}$ & $87_{-6}^{+18}$ & $0.679_{-0.020}^{+0.042}$ & $533_{-16}^{+27}$ & $2.16_{-0.02}^{+0.01}$ & $0.797_{-0.135}^{+0.071}$ & $8.0_{-0.9}^{+1.2}$ & $1.67_{-0.04}^{+0.08}$ \\
\hline 52167.63 & $1276_{-47}^{+96}$ & $814_{-15}^{+72}$ & $564_{-118}^{+51}$ & $94_{-12}^{+11}$ & $0.643_{-0.043}^{+0.039}$ & $551_{-27}^{+32}$ & $2.14_{-0.03}^{+0.03}$ & $0.693_{-0.145}^{+0.087}$ & $8.6_{-0.4}^{+1.4}$ & $1.67_{-0.01}^{+0.09}$ \\
\hline 52168.41 & $1257_{-30}^{+42}$ & $734_{-14}^{+15}$ & $570_{-49}^{+43}$ & $122_{-5}^{+12}$ & $0.661_{-0.031}^{+0.015}$ & $590_{-31}^{+40}$ & $2.15_{-0.02}^{+0.01}$ & $0.777_{-0.068}^{+0.060}$ & $8.2_{-0.6}^{+1.4}$ & $1.66_{-0.05}^{+0.04}$ \\
\hline 52168.48 & $1258_{-53}^{+41}$ & $740_{-38}^{+25}$ & $531_{-51}^{+28}$ & $126_{-13}^{+28}$ & $0.642_{-0.029}^{+0.029}$ & $609_{-37}^{+103}$ & $2.15_{-0.03}^{+0.02}$ & $0.717_{-0.078}^{+0.045}$ & $7.5_{-0.6}^{+0.8}$ & $1.61_{-0.10}^{+0.05}$ \\
\hline 52169.40 & $1282_{-44}^{+44}$ & $734_{-22}^{+16}$ & $568_{-59}^{+72}$ & $139_{-10}^{+13}$ & $0.637_{-0.024}^{+0.024}$ & $601_{-31}^{+46}$ & $2.19_{-0.02}^{+0.02}$ & $0.773_{-0.084}^{+0.099}$ & $8.1_{-0.7}^{+0.4}$ & $1.65_{-0.07}^{+0.01}$ \\
\hline 52169.47 & $1235_{-38}^{+46}$ & $713_{-25}^{+12}$ & $478_{-33}^{+74}$ & $159_{-9}^{+15}$ & $0.634_{-0.023}^{+0.023}$ & $728_{-86}^{+148}$ & $2.16_{-0.03}^{+0.03}$ & $0.671_{-0.052}^{+0.104}$ & $8.2_{-0.7}^{+0.5}$ & $1.65_{-0.07}^{+0.01}$ \\
\hline 52169.54 & $1010_{-68}^{+74}$ & $333_{-44}^{+82}$ & $661_{-111}^{+43}$ & $214_{-11}^{+9}$ & $0.629_{-0.017}^{+0.023}$ & $3921_{-1270}^{+184}$ & $1.96_{-0.02}^{+0.00}$ & $1.983_{-0.423}^{+0.506}$ & $10.0_{-0.2}^{+0.0}$ & $1.66_{-0.05}^{+0.03}$ \\
\hline 52170.25 & $1304_{-32}^{+44}$ & $646_{-20}^{+10}$ & $652_{-97}^{+44}$ & $230_{-18}^{+7}$ & $0.627_{-0.014}^{+0.004}$ & $882_{-51}^{+179}$ & $2.22_{-0.02}^{+0.02}$ & $1.009_{-0.153}^{+0.070}$ & $7.3_{-0.3}^{+0.1}$ & $1.56_{-0.04}^{+0.01}$ \\
\hline 52170.47 & $1292_{-38}^{+62}$ & $681_{-30}^{+18}$ & $562_{-35}^{+90}$ & $235_{-11}^{+13}$ & $0.642_{-0.016}^{+0.016}$ & $1078_{-199}^{+319}$ & $2.22_{-0.04}^{+0.04}$ & $0.824_{-0.062}^{+0.134}$ & $8.0_{-0.7}^{+0.6}$ & $1.63_{-0.07}^{+0.03}$ \\
\hline 52170.60 & $1191_{-110}^{+97}$ & $439_{-43}^{+226}$ & $657_{-61}^{+51}$ & $315_{-16}^{+13}$ & $0.628_{-0.015}^{+0.014}$ & $3545_{-1183}^{+982}$ & $2.14_{-0.03}^{+0.03}$ & $1.495_{-0.202}^{+0.779}$ & $9.2_{-0.5}^{+0.8}$ & $1.67_{-0.01}^{+0.08}$ \\
\hline 52170.81 & $1422_{-114}^{+78}$ & $728_{-35}^{+19}$ & $588_{-30}^{+229}$ & $261_{-18}^{+21}$ & $0.631_{-0.022}^{+0.0018}$ & $1087_{-139}^{+628}$ & $2.28_{-0.06}^{+0.05}$ & $0.808_{-0.056}^{+0.315}$ & $8.7_{-1.0}^{+1.3}$ & $1.66_{-0.05}^{+0.03}$ \\
\hline 52170.87 & $1100_{-130}^{+126}$ & $443_{-100}^{+104}$ & $648_{-64}^{+61}$ & $324_{-15}^{+24}$ & $0.633_{-0.017}^{+0.017}$ & $5171_{-2606}^{+694}$ & $2.12_{-0.04}^{+0.04}$ & $1.463_{-0.360}^{+0.371}$ & $10.0_{-0.9}^{+0.0}$ & $1.68_{-0.07}^{+0.04}$ \\
\hline 52171.53 & $1177_{-105}^{+117}$ & $355_{-71}^{+45}$ & $663_{-46}^{+32}$ & $385_{-14}^{+11}$ & $0.642_{-0.009}^{+0.011}$ & $4516_{-1881}^{+450}$ & $2.15_{-0.03}^{+0.03}$ & $1.869_{-0.395}^{+0.255}$ & $10.0_{-1.2}^{+0.0}$ & $1.72_{-0.08}^{+0.03}$ \\
\hline 52171.66 & $1211_{-87}^{+92}$ & $384_{-61}^{+64}$ & $625_{-30}^{+15}$ & $423_{-9}^{+11}$ & $0.645_{-0.011}^{+0.012}$ & $4245_{-1019}^{+1234}$ & $2.19_{-0.03}^{+0.04}$ & $1.626_{-0.269}^{+0.273}$ & $10.0_{-0.8}^{+0.0}$ & $1.69_{-0.03}^{+0.04}$ \\
\hline 52171.77 & $1259_{-72}^{+55}$ & $334_{-53}^{+28}$ & $681_{-19}^{+69}$ & $477_{-17}^{+19}$ & $0.642_{-0.014}^{+0.013}$ & $4027_{-90}^{+966}$ & $2.23_{-0.05}^{+0.04}$ & $2.036_{-0.329}^{+0.269}$ & $8.7_{-1.3}^{+0.4}$ & $1.66_{-0.10}^{+0.06}$ \\
\hline 52171.90 & $1277_{-67}^{+38}$ & $325_{-23}^{+9}$ & $697_{-68}^{+14}$ & $555_{-5}^{+11}$ & $0.664_{-0.009}^{+0.004}$ & $4996_{-614}^{+435}$ & $2.27_{-0.01}^{+0.01}$ & $2.142_{-0.257}^{+0.071}$ & $9.8_{-1.4}^{+0.2}$ & $1.67_{-0.03}^{+0.02}$ \\
\hline 52172.10 & $1282_{-42}^{+31}$ & $330_{-26}^{+7}$ & $677_{-4}^{+21}$ & $525_{-7}^{+8}$ & $0.657_{-0.007}^{+0.004}$ & $3903_{-1171}^{+1132}$ & $2.26_{-0.01}^{+0.03}$ & $2.050_{-0.163}^{+0.076}$ & $8.8_{-0.7}^{+0.2}$ & $1.66_{-0.06}^{+0.01}$ \\
\hline 52173.10 & $1354_{-29}^{+26}$ & $276_{-29}^{+7}$ & $809_{-14}^{+54}$ & $714_{-6}^{+8}$ & $0.656_{-0.004}^{+0.003}$ & $4981_{-395}^{+1418}$ & $2.35_{-0.02}^{+0.01}$ & $2.929_{-0.314}^{+0.209}$ & $8.9_{-0.5}^{+0.2}$ & $1.66_{-0.04}^{+0.03}$ \\
\hline 52174.30 & $1120_{-39}^{+27}$ & $124_{-8}^{+16}$ & $388_{-5}^{+26}$ & $706_{-8}^{+11}$ & $0.644_{-0.005}^{+0.004}$ & $2614_{-522}^{+2403}$ & $2.26_{-0.05}^{+0.02}$ & $3.129_{-0.197}^{+0.448}$ & $8.5_{-0.9}^{+1.5}$ & $1.65_{-0.09}^{+0.06}$ \\
\hline 52174.77 & $1115_{-48}^{+48}$ & $142_{-61}^{+13}$ & $420_{-41}^{+136}$ & $696_{-20}^{+12}$ & $0.644_{-0.006}^{+0.007}$ & $2783_{-777}^{+2134}$ & $2.28_{-0.07}^{+0.08}$ & $2.952_{-1.307}^{+0.991}$ & $10.0_{-1.2}^{+0.0}$ & $1.69_{-0.03}^{+0.05}$ \\
\hline 52174.91 & $1173_{-82}^{+54}$ & $197_{-45}^{+15}$ & $556_{-26}^{+176}$ & $691_{-10}^{+20}$ & $0.633_{-0.009}^{+0.010}$ & $4450_{-97}^{+1101}$ & $2.31_{-0.04}^{+0.07}$ & $2.826_{-0.660}^{+0.919}$ & $9.4_{-1.8}^{+0.5}$ & $1.66_{-0.14}^{+0.06}$ \\
\hline 52175.15 & $1252_{-61}^{+26}$ & $225_{-13}^{+5}$ & $705_{-3}^{+31}$ & $701_{-5}^{+3}$ & $0.645_{-0.005}^{+0.003}$ & $5012_{-996}^{+155}$ & $2.33_{-0.03}^{+0.01}$ & $3.128_{-0.180}^{+0.156}$ & $9.9_{-0.5}^{+0.1}$ & $1.66_{-0.02}^{+0.02}$ \\
\hline 52176.03 & $1108_{-43}^{+25}$ & $181_{-29}^{+6}$ & $516_{-13}^{+23}$ & $682_{-6}^{+14}$ & $0.630_{-0.001}^{+0.004}$ & $3403_{-758}^{+1699}$ & $2.29_{-0.04}^{+0.03}$ & $2.847_{-0.458}^{+0.161}$ & $10.0_{-0.3}^{+0.0}$ & $1.67_{-0.01}^{+0.03}$ \\
\hline 52176.07 & $1064_{-20}^{+24}$ & $118_{-12}^{+1}$ & $386_{-9}^{+16}$ & $669_{-11}^{+2}$ & $0.633_{-0.002}^{+0.003}$ & $3662_{-365}^{+1390}$ & $2.25_{-0.04}^{+0.02}$ & $3.279_{-0.348}^{+0.142}$ & $10.0_{-0.3}^{+0.0}$ & $1.67_{-0.00}^{+0.02}$ \\
\hline 52176.76 & $1024_{-63}^{+27}$ & $84_{-34}^{+25}$ & $337_{-69}^{+316}$ & $757_{-24}^{+15}$ & $0.622_{-0.006}^{+0.006}$ & $3373_{-102}^{+2574}$ & $2.27_{-0.09}^{+0.11}$ & $4.015_{-1.810}^{+3.954}$ & $10.0_{-1.4}^{+0.0}$ & $1.71_{-0.05}^{+0.03}$ \\
\hline 52177.34 & $1025_{-27}^{+20}$ & $86_{-17}^{+6}$ & $352_{-11}^{+6}$ & $747_{-21}^{+12}$ & $0.623_{-0.001}^{+0.004}$ & $2640_{-0}^{+7360}$ & $2.26_{-0.10}^{+0.04}$ & $4.076_{-0.800}^{+0.303}$ & $10.0_{-0.2}^{+0.0}$ & $1.66_{-0.03}^{+0.03}$ \\
\hline 52178.79 & $1023_{-62}^{+57}$ & $90_{-59}^{+400}$ & $367_{-91}^{+184}$ & $735_{-32}^{+23}$ & $0.602_{-0.009}^{+0.011}$ & $3795_{-1881}^{+2452}$ & $2.30_{-0.11}^{+0.14}$ & $4.053_{-2.813}^{+18.032}$ & $10.0_{-0.8}^{+0.0}$ & $1.60_{-0.07}^{+0.06}$ \\
\hline 52179.71 & $1003_{-60}^{+145}$ & $101_{-28}^{+40}$ & $344_{-152}^{+1313}$ & $746_{-31}^{+24}$ & $0.605_{-0.008}^{+0.008}$ & $2519_{-1191}^{+4702}$ & $2.38_{-0.18}^{+0.15}$ & $3.399_{-1.773}^{+13.063}$ & $10.0_{-2.3}^{+0.0}$ & $1.69_{-0.12}^{+0.08}$ \\
\hline 52179.77 & $1058_{-64}^{+391}$ & $43_{-0}^{+39}$ & $121_{-20}^{+20}$ & $781_{-20}^{+21}$ & $0.590_{-0.011}^{+0.008}$ & $1625_{-502}^{+4172}$ & $2.39_{-0.25}^{+0.28}$ & $2.829_{-0.460}^{+2.611}$ & $10.0_{-2.0}^{+0.0}$ & $1.59_{-0.16}^{+0.09}$ \\
\hline 52179.84 & $969_{-53}^{+57}$ & $65_{-37}^{+7}$ & $295_{-65}^{+297}$ & $756_{-48}^{+57}$ & $0.601_{-0.009}^{+0.010}$ & $2626_{-1261}^{+2869}$ & $2.30_{-0.14}^{+0.17}$ & $4.509_{-2.717}^{+4.563}$ & $10.0_{-0.7}^{+0.0}$ & $1.66_{-0.09}^{+0.07}$ \\
\hline 52179.90 & $999_{-37}^{+572}$ & $50_{-0}^{+24}$ & $348_{-61}^{+903}$ & $743_{-33}^{+35}$ & $0.596_{-0.013}^{+0.011}$ & $4357_{-2911}^{+2856}$ & $2.22_{-0.09}^{+0.27}$ & $6.959_{-1.211}^{+18.362}$ & $8.2_{-1.1}^{+1.8}$ & $1.56_{-0.15}^{+0.11}$ \\
\hline 52179.97 & $1028_{-78}^{+64}$ & $120_{-46}^{+24}$ & $448_{-63}^{+238}$ & $710_{-28}^{+43}$ & $0.598_{-0.013}^{+0.012}$ & $3433_{-1985}^{+4180}$ & $2.36_{-0.13}^{+0.10}$ & $3.720_{-1.504}^{+2.1117}$ & $10.0_{-1.1}^{+0.0}$ & $1.57_{-0.06}^{+0.08}$ \\
\hline 52180.70 & $989_{-69}^{+109}$ & $122_{-34}^{+14}$ & $406_{-113}^{+587}$ & $651_{-24}^{+25}$ & $0.614_{-0.010}^{+0.010}$ & $2619_{-1185}^{+3022}$ & $2.30_{-0.12}^{+0.14}$ & $3.337_{-1.325}^{+4.832}$ & $8.9_{-1.6}^{+1.1}$ & $1.65_{-0.09}^{+0.05}$ \\
\hline 52180.77 & $936_{-52}^{+66}$ & $85_{-41}^{+12}$ & $323_{-39}^{+118}$ & $653_{-24}^{+28}$ & $0.619_{-0.014}^{+0.010}$ & $3789_{-1929}^{+1955}$ & $2.23_{-0.09}^{+0.02}$ & $3.780_{-1.874}^{+1.486}$ & $10.0_{-0.6}^{+0.0}$ & $1.67_{-0.14}^{+0.07}$ \\
\hline 52180.83 & $970_{-77}^{+89}$ & $92_{-63}^{+31}$ & $349_{-32}^{+16}$ & $630_{-25}^{+34}$ & $0.619_{-0.016}^{+0.014}$ & $2559_{-40}^{+3015}$ & $2.24_{-0.09}^{+0.14}$ & $3.808_{-2.639}^{+1.316}$ & $8.0_{-1.1}^{+2.0}$ & $1.56_{-0.17}^{+0.10}$ \\
\hline 52180.90 & $1005_{-109}^{+136}$ & $172_{-62}^{+69}$ & $427_{-24}^{+263}$ & $619_{-26}^{+27}$ & $0.614_{-0.013}^{+0.012}$ & $5010_{-2817}^{+3728}$ & $2.30_{-0.10}^{+0.10}$ & $2.486_{-0.904}^{+1.829}$ & $8.6_{-1.4}^{+1.4}$ & $1.66_{-0.14}^{+0.10}$ \\
\hline 52181.04 & $1001_{-97}^{+151}$ & $132_{-86}^{+60}$ & $343_{-48}^{+193}$ & $609_{-31}^{+38}$ & $0.620_{-0.018}^{+0.014}$ & $2234_{-958}^{+3373}$ & $2.31_{-0.12}^{+0.16}$ & $2.603_{-1.747}^{+1.885}$ & $7.2_{-0.7}^{+2.0}$ & $1.51_{-0.26}^{+0.20}$ \\
\hline 52181.63 & $1116_{-139}^{+625}$ & $72_{-57}^{+48}$ & $905_{-584}^{+877}$ & $694_{-50}^{+45}$ & $0.601_{-0.012}^{+0.017}$ & $1700_{-557}^{+1799}$ & $2.40_{-0.15}^{+0.30}$ & $<120$ & $7.2_{-0.6}^{+0.7}$ & $1.38_{-0.13}^{+0.17}$ \\
\hline 52181.69 & $977_{-49}^{+78}$ & $81_{-24}^{+326}$ & $367_{-217}^{+269}$ & $699_{-22}^{+27}$ & $0.603_{-0.010}^{+0.007}$ & $3696_{-86}^{+1962}$ & $2.30_{-0.18}^{+0.11}$ & $4.533_{-3.002}^{+18.598}$ & $9.9_{-1.8}^{+0.1}$ & $1.66_{-0.14}^{+0.03}$ \\
\hline 52181.76 & $973_{-44}^{+109}$ & $67_{-60}^{+557}$ & $411_{-152}^{+330}$ & $710_{-35}^{+34}$ & $0.597_{-0.014}^{+0.013}$ & $4983_{-0}^{+5017}$ & $2.31_{-0.13}^{+0.17}$ & $6.136_{-5.925}^{+51.298}$ & $8.4_{-1.1}^{+1.6}$ & $1.53_{-0.16}^{+0.14}$ \\
\hline
\end{tabular}


Table 2

(Continued)

\begin{tabular}{|c|c|c|c|c|c|c|c|c|c|c|}
\hline \multirow[t]{2}{*}{ MJD } & \multicolumn{4}{|c|}{ Flux $\left(\times 10^{-10} \mathrm{erg} \mathrm{cm}^{-2} \mathrm{~s}^{-1}\right)$} & \multirow{2}{*}{$\begin{array}{c}\text { Disk Temperature } \\
(\mathrm{keV})\end{array}$} & \multirow{2}{*}{$\begin{array}{c}\text { Ionization } \\
\xi\left(\mathrm{erg} \mathrm{cm} \mathrm{s}^{-1}\right)\end{array}$} & \multirow[t]{2}{*}{$\Gamma$} & \multirow{2}{*}{$\begin{array}{l}\text { Reflection Fraction } \\
\qquad(R)\end{array}$} & \multirow{2}{*}{$\begin{array}{l}\text { Emissivity } \\
\text { Index }(q)\end{array}$} & \multirow{2}{*}{$\begin{array}{c}\text { Disk } \\
\text { Inner Radius (rg) }\end{array}$} \\
\hline & Total $^{\dagger}$ & Power Law ${ }^{\dagger}$ & Reflection $^{\ddagger}$ & $\operatorname{Disk}^{\dagger}$ & & & & & & \\
\hline 52181.83 & $921_{-69}^{+50}$ & $62_{-49}^{+32}$ & $233_{-36}^{+371}$ & $700_{-28}^{+22}$ & $0.607_{-0.009}^{+0.007}$ & $2890_{-1397}^{+3514}$ & $2.29_{-0.14}^{+0.12}$ & $3.768_{-3.049}^{+6.305}$ & $10.0_{-1.9}^{+0.0}$ & $1.69_{-0.07}^{+0.08}$ \\
\hline 52181.89 & $957_{-69}^{+62}$ & $82_{-63}^{+26}$ & $318_{-72}^{+22}$ & $700_{-27}^{+20}$ & $0.605_{-0.007}^{+0.007}$ & $4498_{-2457}^{+2127}$ & $2.30_{-0.11}^{+0.14}$ & $3.896_{-3.124}^{+1.263}$ & $10.0_{-1.6}^{+0.0}$ & $1.71_{-0.13}^{+0.06}$ \\
\hline 52181.96 & $926_{-68}^{+100}$ & $47_{-39}^{+6}$ & $260_{-24}^{+165}$ & $709_{-13}^{+31}$ & $0.605_{-0.010}^{+0.010}$ & $2263_{-1026}^{+1803}$ & $2.24_{-0.15}^{+0.21}$ & $5.535_{-4.633}^{+3.578}$ & $9.4_{-1.9}^{+0.6}$ & $1.64_{-0.14}^{+0.03}$ \\
\hline 52182.39 & $936_{-36}^{+45}$ & $82_{-7}^{+68}$ & $281_{-38}^{+61}$ & $697_{-11}^{+9}$ & $0.605_{-0.003}^{+0.004}$ & $4960_{-2001}^{+1110}$ & $2.28_{-0.07}^{+0.07}$ & $3.451_{-0.545}^{+2.983}$ & $10.0_{-1.1}^{+0.0}$ & $1.70_{-0.04}^{+0.05}$ \\
\hline 52182.49 & $931_{-25}^{+73}$ & $70_{-9}^{+8}$ & $264_{-4}^{+10}$ & $665_{-8}^{+5}$ & $0.615_{-0.003}^{+0.004}$ & $3199_{-456}^{+1828}$ & $2.27_{-0.05}^{+0.03}$ & $3.752_{-0.466}^{+0.435}$ & $10.0_{-0.6}^{+0.0}$ & $1.67_{-0.01}^{+0.04}$ \\
\hline 52183.32 & $948_{-30}^{+19}$ & $86_{-24}^{+6}$ & $289_{-3}^{+10}$ & $654_{-7}^{+4}$ & $0.615_{-0.002}^{+0.003}$ & $3347_{-708}^{+1065}$ & $2.26_{-0.04}^{+0.02}$ & $3.351_{-0.940}^{+0.270}$ & $10.0_{-0.2}^{+0.0}$ & $1.66_{-0.00}^{+0.02}$ \\
\hline 52183.25 & $930_{-37}^{+31}$ & $36_{-17}^{+95}$ & $281_{-12}^{+81}$ & $643_{-9}^{+18}$ & $0.621_{-0.005}^{+0.005}$ & $2347_{-597}^{+1165}$ & $2.26_{-0.07}^{+0.07}$ & $7.878_{-3.706}^{+21.203}$ & $8.1_{-0.8}^{+0.9}$ & $1.63_{-0.07}^{+0.06}$ \\
\hline 52184.16 & $945_{-26}^{+10}$ & $82_{-5}^{+4}$ & $309_{-1}^{+23}$ & $694_{-6}^{+6}$ & $0.614_{-0.002}^{+0.002}$ & $3234_{-532}^{+834}$ & $2.25_{-0.03}^{+0.03}$ & $3.776_{-0.227}^{+0.326}$ & $10.0_{-0.2}^{+0.0}$ & $1.66_{-0.02}^{+0.02}$ \\
\hline 52184.50 & $982_{-42}^{+33}$ & $96_{-40}^{+21}$ & $282_{-14}^{+58}$ & $673_{-13}^{+7}$ & $0.619_{-0.008}^{+0.007}$ & $2867_{-1157}^{+2245}$ & $2.28_{-0.08}^{+0.09}$ & $2.943_{-1.249}^{+0.884}$ & $10.0_{-0.5}^{+0.0}$ & $1.67_{-0.10}^{+0.06}$ \\
\hline 52187.14 & $996_{-30}^{+30}$ & $53_{-24}^{+7}$ & $208_{-10}^{+23}$ & $810_{-12}^{+6}$ & $0.615_{-0.002}^{+0.003}$ & $2634_{-431}^{+541}$ & $2.23_{-0.06}^{+0.04}$ & $3.953_{-1.845}^{+0.671}$ & $10.0_{-0.4}^{+0.0}$ & $1.67_{-0.05}^{+0.03}$ \\
\hline
\end{tabular}

Beginning of disk-dominated high-soft state-power-law flux and consequently reflection fraction poorly contained

\begin{tabular}{|c|c|c|c|c|c|c|c|c|c|c|}
\hline 52190.19 & $1032_{-13}^{+6}$ & $22_{-10}^{+3}$ & $158_{-1}^{+3}$ & $925_{-10}^{+6}$ & $0.613_{-0.001}^{+0.001}$ & $2076_{-184}^{+284}$ & $2.26_{-0.07}^{+0.04}$ & $7.290_{-3.520}^{+0.989}$ & $10.0_{-0.2}^{+0.0}$ & $1.67_{-0.00}^{+0.02}$ \\
\hline 52193.10 & $1059_{-32}^{+73}$ & $10_{-3}^{+4}$ & $329_{-14}^{+971}$ & $928_{-5}^{+4}$ & $0.617_{-0.001}^{+0.001}$ & $2019_{-284}^{+304}$ & $2.51_{-0.12}^{+0.16}$ & $33.626_{-10.345}^{+100.472}$ & $10.0_{-0.3}^{+0.0}$ & $1.67_{-0.00}^{+0.03}$ \\
\hline 52196.08 & $993_{-9}^{+30}$ & $*$ & $251_{-66}^{+1031}$ & $919_{-5}^{+4}$ & $0.610_{-0.001}^{+0.001}$ & $4863_{-2239}^{+1326}$ & $2.48_{-0.06}^{+0.22}$ & * & $10.0_{-0.8}^{+0.0}$ & $1.68_{-0.02}^{+0.06}$ \\
\hline 52199.92 & $1107_{-18}^{*}$ & $*$ & $2929_{-445}^{+4223}$ & $860_{-7}^{+9}$ & $0.591_{-0.002}^{+0.003}$ & $10000_{-1827}^{+0}$ & $2.90_{-0.07}^{+0.17}$ & $*$ & $10.0_{-0.7}^{+0.0}$ & $1.57_{-0.01}^{+0.08}$ \\
\hline 52199.99 & $936_{-25}^{*}$ & $*$ & $512_{-476}^{+59}$ & $851_{-5}^{+17}$ & $0.598_{-0.002}^{+0.002}$ & $3025_{-244}^{+3066}$ & $2.56_{-0.03}^{+0.40}$ & * & $10.0_{-1.6}^{+0.0}$ & $1.67_{-0.05}^{+0.09}$ \\
\hline 52200.05 & $925_{-19}^{*}$ & $*$ & $1172_{-901}^{+7526}$ & $850_{-16}^{+12}$ & $0.598_{-0.002}^{+0.003}$ & $5283_{-2683}^{+4717}$ & $2.52_{-0.08}^{+0.73}$ & $*$ & $10.0_{-2.8}^{+0.0}$ & $1.67_{-0.07}^{+0.12}$ \\
\hline 52202.04 & $871_{-15}^{+10002}$ & $13_{-0}^{+18}$ & $2221_{-1534}^{+1434}$ & $799_{-9}^{+10}$ & $0.597_{-0.002}^{+0.002}$ & $10000_{-2652}^{+0}$ & $2.69_{-0.05}^{+0.18}$ & $170.892_{-118.069}^{+259.681}$ & $9.1_{-0.5}^{+0.9}$ & $64.45_{-31.81}^{+123.12}$ \\
\hline 52202.11 & $866_{-19}^{+10001}$ & $37_{-25}^{+8}$ & $1431_{-149}^{+529}$ & $781_{-7}^{+7}$ & $0.601_{-0.002}^{+0.001}$ & $10000_{-2531}^{+0}$ & $2.76_{-0.10}^{+0.11}$ & $38.362_{-25.591}^{+16.257}$ & $4.0_{-1.0}^{+6.0}$ & $44.45_{-19.45}^{+66.27}$ \\
\hline 52205.42 & $826_{-13}^{+10000}$ & $18_{-0}^{+27}$ & $1596_{-156}^{+3675}$ & $705_{-8}^{+2}$ & $0.587_{-0.003}^{+0.001}$ & $10000_{-2605}^{+0}$ & $2.89_{-0.10}^{+0.11}$ & $87.362_{-8.534}^{+238.209}$ & $10.0_{-1.0}^{+0.0}$ & $1.66_{-0.09}^{+0.06}$ \\
\hline 52211.27 & $701_{-13}^{+10000}$ & $8_{-0}^{+10}$ & $1225_{-256}^{+467}$ & $620_{-8}^{+8}$ & $0.558_{-0.002}^{+0.001}$ & $10000_{-1033}^{+0}$ & $2.93_{-0.05}^{+0.11}$ & $147.234_{-30.751}^{+186.640}$ & $10.0_{-0.4}^{+0.0}$ & $1.67_{-0.05}^{+0.07}$ \\
\hline 52211.39 & $700_{-17}^{*}$ & $*$ & $1357_{-169}^{+671}$ & $628_{-5}^{+13}$ & $0.555_{-0.002}^{+0.002}$ & $10000_{-1994}^{+0}$ & $2.90_{-0.04}^{+0.06}$ & * & $10.0_{-1.2}^{+0.0}$ & $1.68_{-0.03}^{+0.09}$ \\
\hline 52223.57 & $578_{-54}^{+20169}$ & $27_{-0}^{+109}$ & $989_{-179}^{+1975}$ & $488_{-20}^{+21}$ & $0.494_{-0.005}^{+0.005}$ & $10000_{-4858}^{+0}$ & $2.92_{-0.24}^{+0.38}$ & $36.168_{-6.560}^{+160.852}$ & $10.0_{-2.8}^{+0.0}$ & $1.66_{-0.20}^{+0.09}$ \\
\hline 52231.04 & $424_{-25}^{+56}$ & $8_{-0}^{+7}$ & $41_{-24}^{+290}$ & $388_{-34}^{+46}$ & $0.469_{-0.015}^{+0.008}$ & $1351_{-475}^{+1445}$ & $2.16_{-0.22}^{+0.48}$ & $5.162_{-3.030}^{+36.853}$ & $8.6_{-1.5}^{+1.4}$ & $1.54_{-0.29}^{+0.08}$ \\
\hline 52232.04 & $373_{-24}^{+75}$ & $34_{-19}^{+8}$ & $114_{-17}^{+41}$ & $270_{-9}^{+50}$ & $0.512_{-0.028}^{+0.011}$ & $1963_{-884}^{+1836}$ & $2.20_{-0.12}^{+0.19}$ & $3.332_{-1.881}^{+1.396}$ & $8.0_{-1.0}^{+2.0}$ & $1.52_{-0.27}^{+0.13}$ \\
\hline 52233.23 & $335_{-37}^{+39}$ & $60_{-36}^{+31}$ & $150_{-17}^{+23}$ & $176_{-16}^{+16}$ & $0.532_{-0.015}^{+0.018}$ & $4245_{-1358}^{+1780}$ & $2.15_{-0.09}^{+0.09}$ & $2.478_{-1.515}^{+1.312}$ & $10.0_{-2.5}^{+0.0}$ & $1.68_{-0.11}^{+0.07}$ \\
\hline 52234.54 & $276_{-44}^{+80}$ & $115_{-26}^{+92}$ & $124_{-21}^{+51}$ & $67_{-5}^{+15}$ & $0.580_{-0.046}^{+0.024}$ & $2406_{-1319}^{+423}$ & $2.02_{-0.02}^{+0.07}$ & $1.084_{-0.309}^{+0.980}$ & $9.9_{-1.9}^{+0.1}$ & $1.66_{-0.14}^{+0.01}$ \\
\hline 52235.61 & $272_{-52}^{+33}$ & $106_{-31}^{+19}$ & $123_{-33}^{+27}$ & $69_{-11}^{+13}$ & $0.551_{-0.035}^{+0.037}$ & $2839_{-1093}^{+1494}$ & $2.01_{-0.06}^{+0.06}$ & $1.162_{-0.458}^{+0.326}$ & $10.0_{-1.2}^{+0.0}$ & $1.70_{-0.07}^{+0.07}$ \\
\hline 52236.14 & $279_{-25}^{+34}$ & $92_{-25}^{+26}$ & $140_{-52}^{+18}$ & $48_{-8}^{+14}$ & $0.556_{-0.071}^{+0.067}$ & $588_{-76}^{+3803}$ & $2.08_{-0.07}^{+0.07}$ & $\begin{array}{l}1.520_{-0.692}^{+0.466} \\
\end{array}$ & $8.8_{-2.0}^{+1.2}$ & $1.64_{-0.39}^{+0.05}$ \\
\hline 52236.85 & $261_{-17}^{+30}$ & $162_{-12}^{+6}$ & $87_{-4}^{+29}$ & $29_{-4}^{+9}$ & $0.619_{-0.062}^{+0.056}$ & $612_{-89}^{+681}$ & $2.03_{-0.07}^{+0.06}$ & $0.541_{-0.048}^{+0.178}$ & $8.4_{-1.8}^{+1.6}$ & $1.66_{-0.41}^{+0.08}$ \\
\hline 52237.91 & $252_{-14}^{+32}$ & $157_{-17}^{+8}$ & $78_{-7}^{+15}$ & $31_{-7}^{+10}$ & $0.594_{-0.060}^{+0.030}$ & $765_{-201}^{+2223}$ & $1.97_{-0.05}^{+0.05}$ & $0.496_{-0.070}^{+0.101}$ & $8.9_{-1.6}^{+1.1}$ & $1.66_{-0.12}^{+0.06}$ \\
\hline \multicolumn{11}{|c|}{ Return to faint low-hard state where, due to low $\mathrm{S} / \mathrm{N}$, fluxes are not well constrained } \\
\hline 52265.68 & $48_{-14}^{+18}$ & $34_{-5}^{+8}$ & $4_{-2}^{+6}$ & $*$ & $0.951_{-0.851}^{+0.549}$ & $397_{-396}^{+1245}$ & $1.65_{-0.15}^{+0.21}$ & $0.119_{-0.072}^{+0.190}$ & $3.0_{-0.0}^{+7.0}$ & $353.24_{-351.99}^{+46.76}$ \\
\hline 52267.88 & $19_{-7}^{*}$ & $13_{-6}^{+4}$ & $8_{-3}^{+11}$ & $*$ & $0.107_{-0.007}^{+1.393}$ & $345_{-129}^{+3610}$ & $1.83_{-0.34}^{+0.23}$ & $0.605_{-0.388}^{+0.863}$ & $6.0_{-3.0}^{+4.0}$ & $1.68_{-0.43}^{+0.52}$ \\
\hline 52270.00 & $44_{-34}^{+243635}$ & $4_{-0}^{+3}$ & $11_{-8}^{+2373}$ & * & $0.258_{-0.158}^{+0.367}$ & $214_{-213}^{+1550}$ & $2.07_{-0.67}^{+0.50}$ & $3.087_{-2.194}^{+653.620}$ & $10.0_{-7.0}^{+0.0}$ & $1.95_{-0.70}^{+0.93}$ \\
\hline 52273.10 & $7_{-6}^{*}$ & $<7$ & $6_{-3}^{+3}$ & * & $0.728_{-0.628}^{+0.361}$ & $1_{-0}^{+182}$ & $2.94_{-0.62}^{+0.36}$ & $<0.429$ & $10.0_{-5.4}^{+0.0}$ & $1.79_{-0.54}^{+0.35}$ \\
\hline 52277.54 & $*$ & $10_{-5}^{+5}$ & $<7$ & * & $0.105_{-0.005}^{+1.395}$ & $7054_{-7053}^{+2946}$ & $1.49_{-0.09}^{+0.39}$ & $<1.400$ & $4.9_{-1.9}^{+5.1}$ & $3.68_{-2.43}^{+396.32}$ \\
\hline 52279.89 & $17_{-5}^{*}$ & $13_{-4}^{+5}$ & $6_{-4}^{+6}$ & $*$ & $0.100_{-0.000}^{+1.400}$ & $226_{-100}^{+1931}$ & $1.81_{-0.23}^{+0.12}$ & $0.445_{-0.361}^{+0.464}$ & $10.0_{-5.5}^{+0.0}$ & $311.28_{-310.03}^{+88.72}$ \\
\hline 52281.61 & $61_{-46}^{*}$ & $15_{-2}^{+3}$ & $1_{-1}^{+4}$ & * & $0.223_{-0.123}^{+1.070}$ & $787_{-786}^{+9213}$ & $1.77_{-0.33}^{+0.15}$ & $0.068_{-0.067}^{+0.290}$ & $3.0_{-0.0}^{+7.0}$ & $388.54_{-387.29}^{+11.46}$ \\
\hline 52283.75 & $10_{-4}^{*}$ & $<5$ & $>6$ & $*$ & $1.106_{-0.242}^{+0.267}$ & $91_{-87}^{+462}$ & $1.74_{-0.19}^{+0.15}$ & $>1.200$ & $5.9_{-1.8}^{+4.1}$ & $1.51_{-0.26}^{+0.34}$ \\
\hline 52285.00 & $102_{-98}^{*}$ & $<4$ & $<122$ & * & $0.217_{-0.117}^{+1.233}$ & $620_{-396}^{+4938}$ & $1.93_{-0.53}^{+0.87}$ & $*$ & $10.0_{-6.0}^{+0.0}$ & $1.67_{-0.42}^{+0.37}$ \\
\hline 52287.63 & $2_{-1}^{*}$ & $<2$ & $<499$ & * & $1.346_{-1.246}^{+0.154}$ & $340_{-107}^{+1804}$ & $1.79_{-0.39}^{+0.71}$ & $*$ & $10.0_{-5.9}^{+0.0}$ & $268.53_{-267.28}^{+131.47}$ \\
\hline 52291.74 & $>14$ & $12_{-7}^{+14}$ & $<8$ & $*$ & $0.102_{-0.002}^{+1.398}$ & $253_{-252}^{+9747}$ & $1.84_{-0.17}^{+0.20}$ & $<1.6$ & $10.0_{-7.0}^{+0.0}$ & $1.49_{-0.24}^{+1.09}$ \\
\hline 52293.58 & $20_{-4}^{+506}$ & $19_{-4}^{+21}$ & $3_{-1}^{+5}$ & $0_{-0}^{+9}$ & $0.688_{-0.588}^{+0.812}$ & $557_{-343}^{+1376}$ & $1.72_{-0.22}^{+0.16}$ & $0.155_{-0.071}^{+0.297}$ & $4.7_{-1.7}^{+5.3}$ & $344.85_{-343.32}^{+55.15}$ \\
\hline 52295.63 & $17_{-7}^{+689}$ & $14_{-10}^{+3}$ & $4_{-3}^{+4}$ & $0_{-0}^{+168}$ & $0.880_{-0.780}^{+0.282}$ & $538_{-537}^{+4731}$ & $1.64_{-0.24}^{+0.66}$ & $0.260_{-0.267}^{+0.321}$ & $10.0_{-6.5}^{+0.0}$ & $1.67_{-0.42}^{+0.69}$ \\
\hline 52298.03 & $>10$ & $7_{-4}^{+24}$ & $4_{-3}^{+8}$ & $*$ & $0.100_{-0.000}^{+0.163}$ & $911_{-710}^{+1991}$ & $1.63_{-0.23}^{+0.55}$ & $0.624_{-0.568}^{+2.544}$ & $6.1_{-1.9}^{+3.9}$ & $1.31_{-0.06}^{+1.62}$ \\
\hline 52301.07 & $4_{*}^{*}$ & $3_{-1}^{+9}$ & $<4$ & $*$ & $0.469_{-0.369}^{+0.798}$ & $496_{-495}^{+9504}$ & $1.78_{-0.38}^{+0.59}$ & $<2$ & $3.0_{-0.0}^{+7.0}$ & $246.66_{-245.41}^{+153.34}$ \\
\hline 52303.70 & $20_{-6}^{+2690}$ & $12_{-6}^{+4}$ & $4_{-3}^{+9}$ & $<54$ & $0.907_{-0.799}^{+0.563}$ & $1000_{-999}^{+2522}$ & $1.48_{-0.08}^{+0.44}$ & $0.320_{-0.307}^{+0.766}$ & $10.0_{-5.5}^{+0.0}$ & $1.68_{-0.43}^{+0.36}$ \\
\hline 52305.75 & $>17$ & $<6$ & $12_{-7}^{+35}$ & * & $0.109_{-0.009}^{+0.053}$ & $279_{-78}^{+1071}$ & $2.25_{-0.80}^{+0.25}$ & $>0.833$ & $7.8_{-1.4}^{+2.1}$ & $1.31_{-0.06}^{+0.36}$ \\
\hline 52307.08 & $12_{-4}^{+12}$ & $9_{-3}^{+10}$ & $3_{-3}^{+1}$ & $<2$ & $1.235_{-1.135}^{+0.265}$ & $3_{-2}^{+1632}$ & $2.00_{-0.33}^{+0.68}$ & $0.365_{-0.372}^{+0.430}$ & $10.0_{-6.9}^{+0.0}$ & $1.84_{-0.59}^{+0.91}$ \\
\hline
\end{tabular}


Table 2

(Continued)

\begin{tabular}{|c|c|c|c|c|c|c|c|c|c|c|}
\hline \multirow[t]{2}{*}{ MJD } & \multicolumn{4}{|c|}{ Flux $\left(\times 10^{-10} \mathrm{erg} \mathrm{cm}^{-2} \mathrm{~s}^{-1}\right)$} & \multirow{2}{*}{$\begin{array}{c}\text { Disk Temperature } \\
(\mathrm{keV})\end{array}$} & \multirow{2}{*}{$\begin{array}{c}\text { Ionization } \\
\xi\left(\operatorname{erg~cm~s}^{-1}\right)\end{array}$} & \multirow[t]{2}{*}{$\Gamma$} & \multirow{2}{*}{$\begin{array}{l}\text { Reflection Fraction } \\
\qquad(R)\end{array}$} & \multirow{2}{*}{$\begin{array}{l}\text { Emissivity } \\
\text { Index }(q)\end{array}$} & \multirow{2}{*}{$\begin{array}{c}\text { Disk } \\
\text { Inner Radius (rg) }\end{array}$} \\
\hline & Total $^{\dagger}$ & Power Law ${ }^{\dagger}$ & Reflection ${ }^{\ddagger}$ & $\operatorname{Disk}^{\dagger}$ & & & & & & \\
\hline 52309.71 & $11_{-3}^{+1924}$ & $<7$ & $6_{-4}^{+8}$ & $1_{-1}^{+78}$ & $0.625_{-0.525}^{+0.550}$ & $685_{-510}^{+1056}$ & $1.50_{-0.10}^{+0.74}$ & $>0.286$ & $9.5_{-3.8}^{+0.5}$ & $1.56_{-0.31}^{+0.20}$ \\
\hline 52313.61 & $*$ & $<3$ & $<288$ & $*$ & $0.814_{-0.714}^{+0.634}$ & $20_{-19}^{+594}$ & $1.94_{-0.54}^{+0.81}$ & $*$ & $3.0_{-0.0}^{+7.0}$ & $342.12_{-340.86}^{+57.88}$ \\
\hline 52317.57 & $*$ & $<11$ & $2_{-1}^{+1244}$ & $<1003$ & $1.056_{-0.956}^{+0.444}$ & $2_{-1}^{+71}$ & $2.93_{-0.96}^{+0.36}$ & $*$ & $3.6_{-0.6}^{+3.4}$ & $1.32_{-0.07}^{+18.81}$ \\
\hline 52319.11 & $*$ & $*$ & $*$ & $*$ & $*$ & $*$ & $*$ & $*$ & $*$ & $*$ \\
\hline 52321.66 & $12_{*}^{+59}$ & $11_{-5}^{+18}$ & $<4$ & $*$ & $1.474_{-1.374}^{+0.026}$ & $34_{-33}^{+9966}$ & $1.75_{-0.24}^{+0.18}$ & $<0.571$ & $4.6_{-1.6}^{+5.4}$ & $97.99_{-96.74}^{+302.01}$ \\
\hline 52323.78 & $7_{-3}^{*}$ & $*$ & $<11$ & $*$ & $0.396_{-0.296}^{+1.104}$ & $1_{-0}^{+2605}$ & $2.14_{-0.32}^{+0.55}$ & $*$ & $4.2_{-1.2}^{+5.8}$ & $1.58_{-0.33}^{+398.42}$ \\
\hline 52327.37 & $3_{*}^{+345175}$ & $<4$ & $1_{*}^{+36}$ & $*$ & $1.428_{-1.328}^{+0.072}$ & $983_{-982}^{+9017}$ & $1.48_{-0.08}^{+1.82}$ & $*$ & $3.0_{-0.0}^{+7.0}$ & $245.88_{-244.63}^{+154.12}$ \\
\hline 52333.17 & $1_{*}^{+10000}$ & $*$ & $<1$ & $<14196$ & $1.032_{-0.330}^{+0.468}$ & $1_{-0}^{+9771}$ & $1.40_{-0.00}^{+1.90}$ & $*$ & $5.2_{-2.2}^{+4.8}$ & $32.96_{-31.71}^{+367.04}$ \\
\hline 52338.23 & $2_{*}^{+24016}$ & $*$ & $1_{*}^{+5}$ & $*$ & $1.500_{-0.571}^{+0.000}$ & $2_{-1}^{+9998}$ & $1.40_{-0.00}^{+1.90}$ & $*$ & $5.8_{-2.8}^{+4.2}$ & $372.83_{-371.58}^{+27.17}$ \\
\hline 52338.36 & $*$ & $*$ & $*$ & $*$ & * & $*$ & $*$ & $*$ & $*$ & $*$ \\
\hline 52340.06 & $>9$ & $8_{-3}^{+2}$ & $2_{-1}^{+3}$ & $*$ & $0.117_{-0.017}^{+1.383}$ & $965_{-742}^{+6466}$ & $1.79_{-0.14}^{+0.28}$ & $0.211_{-0.192}^{+0.432}$ & $10.0_{-7.0}^{+0.0}$ & $2.16_{-0.91}^{+397.84}$ \\
\hline 52344.98 & $*$ & $1_{*}^{+2}$ & $2_{*}^{+70}$ & $*$ & $0.100_{-0.000}^{+1.400}$ & $2177_{-2176}^{+7823}$ & $1.40_{-0.00}^{+0.65}$ & $*$ & $10.0_{-7.0}^{+0.0}$ & $1.73_{-0.48}^{+398.27}$ \\
\hline 52349.02 & $2_{-1}^{+10000}$ & $<10000$ & $*$ & $<7$ & $0.736_{-0.318}^{+0.764}$ & $257_{-256}^{+590}$ & $1.40_{-0.00}^{+0.84}$ & $*$ & $9.8_{-6.8}^{+0.2}$ & $196.40_{-195.15}^{+203.60}$ \\
\hline 52358.59 & $6_{-3}^{+5}$ & $4_{-3}^{+1}$ & $2_{-1}^{+12}$ & $0_{-0}^{+30026}$ & $1.500_{-1.400}^{+0.000}$ & $503_{-502}^{+4011}$ & $\begin{array}{c}1.50_{-0.10}^{+0.73}\end{array}$ & $0.494_{-0.549}^{+3.508}$ & $3.0_{-0.0}^{+7.0}$ & $377.39_{-376.14}^{+22.61}$ \\
\hline 52360.77 & $>6$ & $4_{-1}^{+1}$ & $<7$ & $*$ & $0.101_{-0.001}^{+0.014}$ & $614_{-227}^{+3437}$ & $1.85_{-0.45}^{+0.34}$ & $<2.333$ & $10.0_{-5.1}^{+0.0}$ & $1.68_{-0.43}^{+0.22}$ \\
\hline 52423.09 & $*$ & $*$ & $*$ & $*$ & $*$ & $*$ & $*$ & $*$ & $*$ & $*$ \\
\hline 52445.85 & $*$ & $*$ & $*$ & $*$ & $*$ & $*$ & $*$ & $*$ & $*$ & $*$ \\
\hline
\end{tabular}

Notes.

*Error or parameter could not be constrained.

${ }^{\dagger}$ Extrapolated flux in the $0.1-1000 \mathrm{keV}$ range.

${ }^{\ddagger}$ Extrapolated flux in the $1 \mathrm{ev}-1000 \mathrm{keV}$ range.

3 . The $F_{\text {reflection }}-F_{\text {powerlaw }}$ plane for the periods covering the hard-intermediate to the soft-intermediate spectral states displays two distinct behaviors. Early in the outburst, during the HIS, this plane is nearly flat (slope of $-0.24 \pm 0.02$ ) with the power-law flux varying by $\sim 5 \times$ and the corresponding reflection flux by $\sim 1.5 \times$. During the SIS that followed, this behavior becomes distinctively different with an $F_{\text {reflection }}-F_{\text {powerlaw }}$ slope of $2.7 \pm 0.1$.

4. The nearly flat behavior of the $F_{\text {reflection }}-F_{\text {powerlaw }}$ plane seen for the HIS cannot be explained away by variations in the ionization of the accretion disk and we propose that the most likely explanation is that of the light-bending model of Miniutti \& Fabian (2004).

5. The HIS-SIS transition is accompanied by a sharp increase in the reflection fraction, which we interpret as a sudden collapse of the corona as the system approaches the thermal state. The collapsed corona now experiences stronger effects of light bending due to its proximity to the black hole. The radiation from the corona is focused toward the disk thus systematically increasing the reflection and decreasing the continuum.

6 . We confirm the $R-\Gamma$ correlation during the LHS and early HIS but find that this relation does not hold once the system has transited into the SIS.

7. We find a strong linear correlation between the reflection fraction and the coherence of the HF QPOs. We also find a correlation between the frequency of the QPO and $R$, which is well explained with a simple log-linear relation.

8. We find a strong correlation between the frequency of the QPO and the ionization state of the accretion disk. This relationship is suggestive of an intrinsic relationship between the irradiation of the disk and its magnetic field properties.
9. We have presented a scenario within the collapsing corona toy model where the increase in the coherence of QPO with increasing reflection fraction is a consequence of decreasing emitting region as the corona collapse to regions closer to the black hole where light bending significantly increases $R$. Similarly, as the corona collapses, the increase in the QPO frequency could be associated with a decrease in the radial extent of the corona.

10. In the LHS and HIS, the radio-flux density varies by a factor of $\sim 5$ with no change in the reflection fraction. The "ballistic" radio ejection associated with the HIS-SIS transition is accompanied by a sharp increase in the reflection fraction.

11. We show that the HIS-SIS transition is not due to variations in the inner radius of the accretion disk which is found to be stable at $1.65 \pm 0.08 r_{\mathrm{g}}$. Assuming that this is the radius of the innermost stable circular orbit, we find a spin of $a \gtrsim 0.96$ in excellent agreement with values measured in other works.

12. Our work shows that the configuration of the corona-and possibly that of the magnetic field-is instrumental in defining the state of the system.

R.C.R. thanks the Michigan Society of Fellows and NASA. R.C.R. is supported by NASA through the Einstein Fellowship Program, grant number PF1-120087 and is a member of the Michigan Society of Fellows. A.C.F. thanks the Royal Society.

\section{REFERENCES}

Anders, E., \& Grevesse, N. 1989, GeCoA, 53, 197

Arnaud, K. A. 1996, in ASP Conf. Ser. 101, Astronomical Data Analysis Software and Systems V, ed. G. H. Jacoby \& J. Barnes (San Francisco, CA: ASP), 17

Ballantyne, D. R., McDuffie, J. R., \& Rusin, J. S. 2011, ApJ, 734, 112 
Ballantyne, D. R., Ross, R. R., \& Fabian, A. C. 2001, MNRAS, 327, 10 Ballantyne, D. R., Vaughan, S., \& Fabian, A. C. 2003, MNRAS, 342, 239 Balucinska-Church, M., \& McCammon, D. 1992, ApJ, 400, 699 Bardeen, J. M., Press, W. H., \& Teukolsky, S. A. 1972, ApJ, 178, 347 Barret, D., Olive, J.-F., \& Miller, M. C. 2006, MNRAS, 370, 1140 Barret, D., Olive, J.-F., \& Miller, M. C. 2007, MNRAS, 376, 1139 Beckwith, K., \& Done, C. 2004, MNRAS, 352, 353

Belloni, T. M. (ed.) 2010, The Jet Paradigm (Lecture Notes in Physics, Vol. 794; Berlin: Springer), 53

Beloborodov, A. M. 1999a, in ASP Conf. Ser. 161, High Energy Processes in Accreting Black Holes, ed. J. Poutanen \& R. Svensson (San Francisco, CA: ASP), 295

Beloborodov, A. M. 1999b, ApJL, 510, 123

Brenneman, L. W., \& Reynolds, C. S. 2006, ApJ, 652, 1028

Brenneman, L. W., Reynolds, C. S., Nowak, M. A., et al. 2011, ApJ, 736, 103

Cackett, E. M., Miller, J. M., Ballantyne, D. R., et al. 2010, ApJ, 720, 205

Cackett, E. M., Miller, J. M., Bhattacharyya, S., et al. 2008, ApJ, 674, 415

Chartas, G., Kochanek, C. S., Dai, X., Poindexter, S., \& Garmire, G. 2009, ApJ, 693, 174

Chartas, G., Kochanek, C. S., Dai, X., et al. 2012, ApJ, 757, 137

Corbel, S., Fender, R. P., Tomsick, J. A., Tzioumis, A. K., \& Tingay, S. 2004, ApJ, 617, 1272

Cui, W., Zhang, S. N., \& Chen, W. 1998, ApJL, 492, 53

Dai, X., Kochanek, C. S., Chartas, G., et al. 2010, ApJ, 709, 278

Dauser, T., Wilms, J., Reynolds, C. S., \& Brenneman, L. W. 2010, MNRAS, 409, 1534

Demleitner, M., Accomazzi, A., Eichhorn, G., et al. 2001, in ASP Conf. Ser. 238, Astronomical Data Analysis Software and Systems X, ed. F. R. Harnden, Jr., F. A. Primini, \& H. E. Payne (San Francisco, CA: ASP), 321

di Salvo, T., D’Aí, A., Iaria, R., et al. 2009, MNRAS, 398, 2022

di Salvo, T., Iaria, R., Méndez, M., et al. 2005, ApJL, 623, 121

Done, C. 2002, RSPTA, 360, 1967

Dovčiak, M., Karas, V., \& Yaqoob, T. 2004, ApJS, 153, 205

Dunn, R. J. H., Fender, R. P., Körding, E. G., Belloni, T., \& Cabanac, C. 2010, MNRAS, 403, 61

Dunn, R. J. H., Fender, R. P., Körding, E. G., Belloni, T., \& Merloni, A. 2011, MNRAS, 411, 337

Ebisawa, K., Ogawa, M., Aoki, T., et al. 1994, PASJ, 46, 375

Fabian, A. C., \& Ross, R. R. 2010, SSRv, 157, 167

Fabian, A. C., \& Vaughan, S. 2003, MNRAS, 340, L28

Fabian, A. C., Vaughan, S., Nandra, K., et al. 2002, MNRAS, 335, L1

Fabian, A. C., Wilkins, D. R., Miller, J. M., et al. 2012a, MNRAS, 424, 217

Fabian, A. C., Zoghbi, A., Ross, R. R., et al. 2009, Natur, 459, 540

Fabian, A. C., Zoghbi, A., Wilkins, D., et al. 2012b, MNRAS, 419, 116

Falcke, H., Körding, E., \& Markoff, S. 2004, A\&A, 414, 895

Fender, R. P. 2001, MNRAS, 322, 31

Fender, R. P., Belloni, T. M., \& Gallo, E. 2004, MNRAS, 355, 1105

Fender, R. P., Homan, J., \& Belloni, T. M. 2009, MNRAS, 396, 1370

Foreman-Mackey, D., Hogg, D. W., Lang, D., \& Goodman, J. 2012, arXiv: 1202.3665

Gallo, E., Fender, R. P., \& Pooley, G. G. 2003, MNRAS, 344, 60

García, J., \& Kallman, T. R. 2010, ApJ, 718, 695

García, J., Kallman, T. R., \& Mushotzky, R. F. 2011, ApJ, 731, 131

George, I. M., \& Fabian, A. C. 1991, MNRAS, 249, 352

Gilfanov, M., Churazov, E., \& Revnivtsev, M. 1999, A\&A, 352, 182

Gilfanov, M., Churazov, E., \& Revnivtsev, M. 2000, MNRAS, 316, 923

Hiemstra, B., Méndez, M., Done, C., et al. 2011, MNRAS, 411, 137

Homan, J., Klein-Wolt, M., Rossi, S., et al. 2003, ApJ, 586, 1262

Ibragimov, A., Poutanen, J., Gilfanov, M., Zdziarski, A. A., \& Shrader, C. R. 2005, MNRAS, 362, 1435

King, A. L., Miller, J. M., Raymond, J., et al. 2012, ApJL, 746, 20

Laor, A. 1991, ApJ, 376, 90

Larsson, J., Fabian, A. C., Miniutti, G., \& Ross, R. R. 2007, MNRAS, 376, 348

Lightman, A. P., \& White, T. R. 1988, ApJ, 335, 57

Magdziarz, P., \& Zdziarski, A. A. 1995, MNRAS, 273, 837

Malzac, J., Beloborodov, A. M., \& Poutanen, J. 2001, MNRAS, 326, 417

Markoff, S., Nowak, M. A., \& Wilms, J. 2005, ApJ, 635, 1203

Martocchia, A., Karas, V., \& Matt, G. 2000, MNRAS, 312, 817

Martocchia, A., \& Matt, G. 1996, MNRAS, 282, L53

Matt, G., Fabian, A. C., \& Ross, R. R. 1993, MNRAS, 262, 179

Matt, G., Fabian, A. C., \& Ross, R. R. 1996, MNRAS, 278, 1111

Matt, G., Perola, G. C., \& Piro, L. 1991, A\&A, 247, 25

McKinney, J. C., Tchekhovskoy, A., \& Blandford, R. D. 2012, MNRAS, 423, 3083

Merloni, A., Heinz, S., \& di Matteo, T. 2003, MNRAS, 345, 1057
Miller, J. M. 2007, ARA\&A, 45, 441

Miller, J. M., Cackett, E. M., \& Reis, R. C. 2009a, ApJL, 707, 77

Miller, J. M., Fabian, A. C., Wijnands, R., et al. 2002, ApJL, 570, 69

Miller, J. M., Homan, J., \& Miniutti, G. 2006a, ApJL, 652, 113

Miller, J. M., Homan, J., Steeghs, D., et al. 2006b, ApJ, 653, 525

Miller, J. M., Pooley, G. G., Fabian, A. C., et al. 2012, arXiv e-prints

Miller, J. M., Raymond, J., Reynolds, C. S., et al. 2008a, ApJ, 680, 1359

Miller, J. M., Reynolds, C. S., Fabian, A. C., Miniutti, G., \& Gallo, L. C. 2009b, ApJ, 697, 900

Miller, J. M., Reynolds, C. S., Fabian, A. C., et al. 2008b, ApJL, 679, 113

Miniutti, G., \& Fabian, A. C. 2004, MNRAS, 349, 1435

Miniutti, G., Fabian, A. C., Goyder, R., \& Lasenby, A. N. 2003, MNRAS, 344, L22

Miniutti, G., Fabian, A. C., \& Miller, J. M. 2004, MNRAS, 351, 466

Molina, M., Bassani, L., Malizia, A., et al. 2009, MNRAS, 399, 1293

Morgan, C. W., Hainline, L. J., Chen, B., et al. 2012, arXiv e-prints

Nandra, K., George, I. M., Mushotzky, R. F., Turner, T. J., \& Yaqoob, T. 1997, ApJ, 477, 602

Nandra, K., O'Neill, P. M., George, I. M., \& Reeves, J. N. 2007, MNRAS, 382,194

Nayakshin, S., \& Kallman, T. R. 2001, ApJ, 546, 406

Nayakshin, S., Kazanas, D., \& Kallman, T. R. 2000, ApJ, 537, 833

Neilsen, J., \& Lee, J. C. 2009, Natur, 458, 481

Niedźwiecki, A., \& Życki, P. T. 2008, MNRAS, 386, 759

Nowak, M. A., Wagoner, R. V., Begelman, M. C., \& Lehr, D. E. 1997, ApJL, 477,91

Nowak, M. A., Wilms, J., \& Dove, J. B. 2002, MNRAS, 332, 856

Oishi, J. S., \& Mac Low, M.-M. 2011, ApJ, 740, 18

O’Neill, S. M., Reynolds, C. S., Miller, M. C., \& Sorathia, K. A. 2011, ApJ, 736, 107

Orosz, J. A., McClintock, J. E., Remillard, R. A., \& Corbel, S. 2004, ApJ, 616,376

Ponti, G., Fender, R. P., Begelman, M. C., et al. 2012, MNRAS, 422, L11

Poutanen, J., Krolik, J. H., \& Ryde, F. 1997, MNRAS, 292, L21

Psaltis, D., Belloni, T., \& van der Klis, M. 1999, ApJ, 520, 262

Reis, R. C., Fabian, A. C., \& Miller, J. M. 2010, MNRAS, 402, 836

Reis, R. C., Fabian, A. C., Ross, R. R., \& Miller, J. M. 2009a, MNRAS, 395, 1257

Reis, R. C., Fabian, A. C., Ross, R. R., et al. 2008, MNRAS, 387, 1489

Reis, R. C., Fabian, A. C., \& Young, A. J. 2009b, MNRAS, 399, L1

Reis, R. C., Miller, J. M., \& Fabian, A. C. 2009c, MNRAS, 395, L52

Reis, R. C., Miller, J. M., Reynolds, M. T., Fabian, A. C., \& Walton, D. J. 2012, ApJ, 751, 34

Remillard, R. 2001, IAU Circ., 7707, 1

Remillard, R. A., \& McClintock, J. E. 2006, ARA\&A, 44, 49

Reynolds, C. S., Brenneman, L. W., Lohfink, A. M., et al. 2012, ApJ, submitted (arXiv:1204.5747)

Reynolds, M., \& Miller, J. 2011, arXiv:1112.2249

Ross, R. R., \& Fabian, A. C. 1993, MNRAS, 261, 74

Ross, R. R., \& Fabian, A. C. 2005, MNRAS, 358, 211

Ross, R. R., \& Fabian, A. C. 2007, MNRAS, 381, 1697

Ross, R. R., Fabian, A. C., \& Young, A. J. 1999, MNRAS, 306, 461

Rossi, S., Homan, J., Miller, J. M., \& Belloni, T. 2005, MNRAS, 360, 763

Shapiro, S. L., Lightman, A. P., \& Eardley, D. M. 1976, ApJ, 204, 187

Steiner, J. F., \& McClintock, J. E. 2012, ApJ, 745, 136

Steiner, J. F., Reis, R. C., McClintock, J. E., et al. 2011, MNRAS, 416, 941

Stella, L., Vietri, M., \& Morsink, S. M. 1999, ApJL, 524, 63

Sunyaev, R. A., \& Titarchuk, L. G. 1980, A\&A, 86, 121

Tanaka, Y., Nandra, K., Fabian, A. C., et al. 1995, Natur, 375, 659

Tomsick, J. A., Yamaoka, K., Corbel, S., et al. 2009, ApJL, 707, 87

Ueda, Y., Ebisawa, K., \& Done, C. 1994, PASJ, 46, 107

Walton, D. J., Reis, R. C., Cackett, E. M., Fabian, A. C., \& Miller, J. M. 2012, MNRAS, 422, 2510

Wilkins, D. R., \& Fabian, A. C. 2011, MNRAS, 414, 1269

Wilkins, D. R., \& Fabian, A. C. 2012, MNRAS, 424, 1284

Wilms, J., Nowak, M. A., Pottschmidt, K., Pooley, G. G., \& Fritz, S. 2006, A\&A, 447,245

Yan, L.-H., \& Wang, J.-C. 2012, RAA, 12, 269

Yungelson, L. R., Lasota, J.-P., Nelemans, G., et al. 2006, A\&A, 454, 559

Zdziarski, A. A., Lubiński, P., Gilfanov, M., \& Revnivtsev, M. 2003, MNRAS, 342,355

Zdziarski, A. A., Lubiński, P., \& Smith, D. A. 1999, MNRAS, 303, L11

Zycki, P. T., Krolik, J. H., Zdziarski, A. A., \& Kallman, T. R. 1994, ApJ, 437, 597 